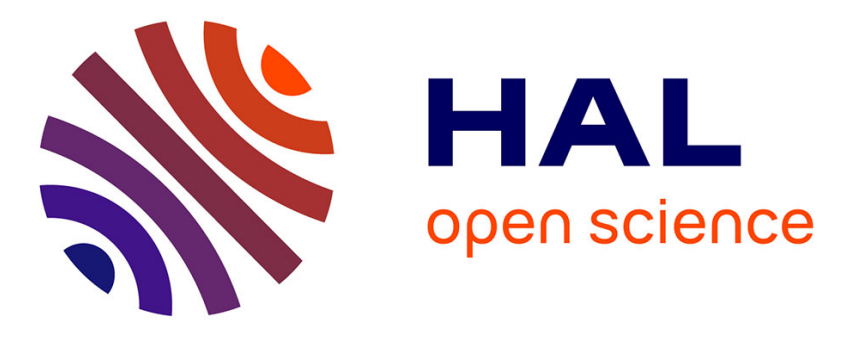

\title{
Electron dynamics and cross-shock potential at the quasi-perpendicular Earth's bow shock
}

Bertrand Lefebvre, Steven J Schwartz, Andrew F Fazakerley, Pierrette Décréau

\section{- To cite this version:}

Bertrand Lefebvre, Steven J Schwartz, Andrew F Fazakerley, Pierrette Décréau. Electron dynamics and cross-shock potential at the quasi-perpendicular Earth's bow shock. Journal of Geophysical Research Space Physics, 2007, 112, pp.9212 - 9212. 10.1029/2007JA012277 . insu-01408851

\section{HAL Id: insu-01408851 https://hal-insu.archives-ouvertes.fr/insu-01408851}

Submitted on 5 Dec 2016

HAL is a multi-disciplinary open access archive for the deposit and dissemination of scientific research documents, whether they are published or not. The documents may come from teaching and research institutions in France or abroad, or from public or private research centers.
L'archive ouverte pluridisciplinaire $\mathbf{H A L}$, est destinée au dépôt et à la diffusion de documents scientifiques de niveau recherche, publiés ou non, émanant des établissements d'enseignement et de recherche français ou étrangers, des laboratoires publics ou privés. 


\title{
Electron dynamics and cross-shock potential at the quasi-perpendicular Earth's bow shock
}

\author{
Bertrand Lefebvre, ${ }^{1}$ Steven J. Schwartz, ${ }^{1}$ Andrew F. Fazakerley, ${ }^{2}$ and Pierrette Décréau ${ }^{3}$ \\ Received 16 January 2007; revised 8 May 2007; accepted 22 June 2007; published 18 September 2007.
}

[1] The evolution of the electron distribution function through quasi-perpendicular collisionless shocks is believed to be dominated by the electron dynamics in the large-scale coherent and quasi-stationary magnetic and electric fields. We investigate the electron distributions measured on board Cluster by the Plasma Electron and Current Experiment (PEACE) instrument during three quasi-perpendicular bow shock crossings. Observed distributions are compared with those predicted by electron dynamics resulting from conservation of the first adiabatic invariant and energy in the de Hoffmann-Teller frame, for all pitch angles and all types of trajectories (passing and, for the first time, reflected or trapped). The predicted downstream velocity distributions are mapped from upstream measurements using an improved Liouville mapping technique taking into account the overshoots. Furthermore, for one of these crossings we could take advantage of the configuration of the Cluster quartet to compare mapped upstream velocity distributions with those simultaneously measured at a relatively well magnetically connected downstream location. Consequences of energy and adiabatic invariant conservation are found to be compatible with the observed electron distributions, confirming the validity of electron "heating" theories based on DC fields as zeroth-order approximations, but some systematic deviations are found between the dynamics of low- and high-adiabatic invariant electrons. Our approach also provides a way to estimate the cross-shock electric potential profile making full use of the electron measurements, and the results are compared to other estimates relying on the steady state dissipationless electron fluid equations. At the temporal resolution of the instruments, the scales associated to the change of the potential generally appear to be comparable to those of the magnetic field, but some differences between the methods appear within the shock transition. It is argued that potentials evaluated from Liouville mapping rely on less restrictive hypotheses and are therefore more reliable. Finally, we show how, in contrast to methods using electron velocity moments, the technique can be used to produce high-time-resolution electric potentials and discuss the electric potential profiles through the shock.

Citation: Lefebvre, B., S. J. Schwartz, A. F. Fazakerley, and P. Décréau (2007), Electron dynamics and cross-shock potential at the quasi-perpendicular Earth's bow shock, J. Geophys. Res., 112, A09212, doi:10.1029/2007JA012277.

\section{Introduction}

[2] The electric cross-shock potential at collisionless quasi-perpendicular shocks controls in great part the deceleration of the bulk of the incoming ions, electron heating and some particle acceleration mechanisms [e.g., Lee et al., 1996; Zank et al., 1996]. The potential difference between the two sides of the shock and the scales over which it varies are therefore of considerable interest to understand

\footnotetext{
${ }^{1}$ Blackett Laboratory, Imperial College London, London, UK.

${ }^{2}$ Mullard Space Science Laboratory, University College London, London, UK.

${ }^{3}$ LPCE, CNRS and University of Orléans, Orléans, France.

Copyright 2007 by the American Geophysical Union. 0148-0227/07/2007JA012277
}

the processes controlling the shock and the associated particle dynamics.

[3] Electrons thanks to their small inertia provide good tracers of field variations through a shock, and Feldman et al. [1983] have pointed out the possible signature of the cross-shock potential in the formation of an electron "beam" observed during some shock crossings. Goodrich and Scudder [1984] have taken this idea further proposing a mechanism by which in the drift approximation the largescale magnetic field and cross-shock electric potential shape the bulk of electron velocity distribution. For instance, this mechanism shows how the frame-dependence of the potential (which is related to the noncoplanar component of the magnetic field inside the shock layer) can explain the relatively small heating of electrons compared to ions at moderate Mach number shocks. It also gives some hints as to why the electron heating at weak quasi-perpendicular 
shocks is predominantly perpendicular and more isotropic at stronger shocks (see Scudder [1995] for a review). This effect was shown to account quite well for the electron heating and gross shape of the electron distribution observed at some crossings of the Earth's bow shock [Scudder et al., 1986b; Hull et al., 1998] and interplanetary shocks [Fitzenreiter et al., 2003], suggesting that in conditions typical of these shocks it dominates the anomalous heating by small-scale turbulence invoked in early theories of collisionless shocks [Sagdeev, 1966]. However, it was suggested by Balikhin et al. [1993; see also Balikhin et al., 1998] that possible short-scale (of the order of the electron inertial length $c / \omega_{p e}$ ) electric field bursts in the shock ramp could invalidate the drift approximation by locally demagnetizing the electrons, thereby possibly heating them more strongly. Such large-amplitude small-scale electric bursts were recently observed by Walker et al. [2004] at a few marginally supercritical quasi-perpendicular shock crossings using high-resolution electric field measurements on board Cluster.

[4] Although multisatellite missions have allowed considerable progress on the knowledge of shock scales (see Bale et al. [2005] for a review of recent Cluster results), those associated to the cross-shock potential remain less well known. A major reason for that is the difficulty to estimate the potential directly from electric field measurements, mainly due to the necessity to estimate the electric field in the appropriate reference frame, the satellite's wake effects and, on many satellites, the availability of only two components of the electric field. Few direct approaches of this kind have appeared in the literature [Formisano, 1982; Wygant et al., 1987; Walker et al., 2004], and alternative methods have to be sought.

[5] In this paper we investigate electron dynamics and its relationship to the cross-shock potential for three quasiperpendicular shock crossings by the Cluster satellites. The method of characteristics (Liouville mapping) which relates the upstream and downstream velocity distributions is reviewed in section 2, paying particular attention to the effect of nonmonotonic magnetic and potential profiles. After a general description of the data set (section 3) and the shocks (section 5), the predictions of this technique are checked in detail by comparing mapped distributions to the observed ones downstream and within the shock layer, slightly beyond the ramp. This static view is completed by a more dynamical picture comparing electron guiding center trajectories to contours of the electron distribution function (section 5). Beyond the confirmation that observations are essentially consistent with the prediction of the Liouville mapping, some discrepencies depending on electron magnetic moment are pointed out. In section 6 we show how, taking the assumptions of Goodrich and Scudder [1984] and making use of the Liouville mapping, an estimate of electric cross-shock potential can be retrieved from measurements of electron velocity distributions. This result is compared with others relying on dissipationless electron fluid equations [Scudder et al., 1986b; Hull et al., $2000]$ and to magnetic field profiles. The technique can also be restricted to data from electrons within a particular range of adiabatic invariants, which suggests in a manner consistent with the results from section 5 that depending on their magnetic moments electrons can be mapped in a slightly different way across the shock. Finally, it is shown in the same section how in some cases only partial measurements of the velocity distribution can be used to estimate the electric potential at a higher temporal resolution.

\section{Liouville Mapping and the Cross-Shock Potential}

\subsection{Liouville Mapping}

[6] A fundamental property of the Vlasov equation is that it conserves the phase space density along its characteristics, which correspond to the particle trajectories. In the drift approximation, a drift-kinetic equation can be constructed which retains this property, the trajectories being those of the particle guiding centers [Brizard and Hahm, 2007]. This property can be used to map part of the phase space density from one location to another, at phase space coordinates which are connected by particle trajectories. The technique, diversely known as the Liouville mapping, Vlasov mapping, or method of characteristics, requires that particle trajectories can be estimated with sufficient accuracy, which requires in particular some knowledge of the fields (see Schwartz et al. [2000] and Hull et al. [2000] for thorough discussions of the experimental limitations of the technique). Furthermore, for the method to be useful the trajectories connecting the two locations to be mapped shall occupy a significant enough volume in phase space in order to to map a significant fraction of the velocity distribution.

[7] Goodrich and Scudder [1984] suggested that this technique could be used to map the thermal part of the phase space measured at supercritical quasi-perpendicular shocks using the large-scale (DC) magnetic field measured on board spacecraft, under the assumption of shock planarity and stationarity. Furthermore, particle trajectories are greatly simplified in the drift approximation, assuming (1) gyrotropy of the electron distribution function and conservation of the magnetic moment or first adiabatic invariant $\mu=m v_{\perp}^{2} / 2 B$ (where $v_{\perp}$ is the gyration velocity) and (2) conservation of electron energy in the de HoffmannTeller frame, the shock rest frame where the asymptotic upstream and downstream fluid velocities are parallel to the magnetic field. This energy, conserved to the first order in the ratio of the electron gyroradius to the gradient characteristic scale, is

$$
\begin{aligned}
\mathcal{E}^{\prime} & =\frac{1}{2} m v_{\|}^{\prime 2}+\mu B-e \phi^{\prime} \\
& =\frac{1}{2} m v_{\|}^{\prime 2}+\hat{U}^{\prime} \\
& =\text { const }
\end{aligned}
$$

where the prime denotes quantities in the de HoffmannTeller frame and $\phi^{\prime}$ is the electric potential in this frame [Goodrich and Scudder, 1984; Scudder, 1995]. While by definition the $\mathbf{E} \times \mathbf{B}$ drifts vanish away from the shock front in the de Hoffmann-Teller frame, they are assumed to be negligible in the above expression through the shock transition, together with the other guiding-center drifts. These hypotheses leave only one degree of freedom for the guiding-center motion in the effective potential $\hat{U}^{\prime}$, which easily determines the velocity of an electron as a function of its initial velocity and the fields at the initial and current 

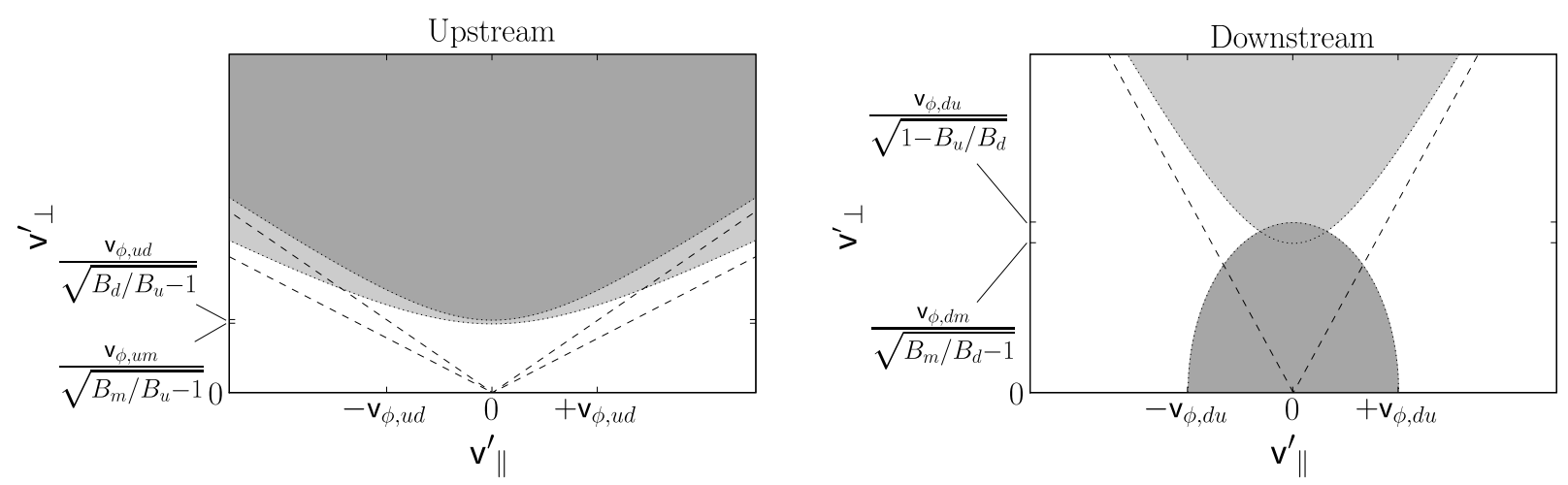

Figure 1. Regions of passing (white) and nonpassing (grey) electron trajectories in HT velocity space in the presence of an overshoot, (left) upstream and (right) downstream. With respect to a monotonic shock profile, the presence of a magnetic and potential overshoot results in new sets of nonpassing (i.e., reflected or trapped) trajectories, shown in lighter grey. Downstream, this reduces the domain of the Liouville-mapped distribution function particularly in the perpendicular direction (right). The dashed lines on the left figure have slopes $\pm\left(B_{m} / B_{u}-1\right)^{-1 / 2}$ and $\pm\left(B_{d} / B_{u}-1\right)^{-1 / 2}$, and on the right-hand figure $\pm\left(B_{m} / B_{d}-1\right)^{-1 / 2}$. The two perpendicular velocities labelled on each plot are equal when $\Delta \phi \propto$ $\Delta B$. The two figures have the same scales, and parameters correspond to the 19 December 2003 shock.

locations. For a planar stationary shock, and assuming that the fields vary monotonically, the velocity distribution function at an abscissa $x_{i}$ along the shock normal is thus related to the one at an arbitrary reference location $x_{j}$ by

$$
\begin{aligned}
f\left(x_{i}, v_{\|, i}^{\prime}, v_{\perp, i}^{\prime}\right)= & f\left(x_{j}, \operatorname{sgn}\left(v_{\|, i}^{\prime}\right)\left[v_{\|, i}^{\prime 2}+\left(1-b_{i j}\right) v_{\perp, i}^{\prime 2}\right.\right. \\
& \left.\left.+\sigma_{i j} v_{\phi, i j}^{\prime 2}\right]^{1 / 2}, b_{i j}^{1 / 2} v_{\perp, i}^{\prime}\right)
\end{aligned}
$$

with $b_{i j}=B_{j} / B_{i}, \sigma_{i j}=\operatorname{sgn}\left(\phi_{j}^{\prime}-\phi_{i}^{\prime}\right)$ and $v_{\phi, i j}^{\prime 2}=2 e\left|\phi_{j}^{\prime}-\phi_{i}^{\prime}\right| / m$. This equality is true only for phase space loci which are connected by particle trajectories, which restricts it to velocities satisfying

$$
v_{\|, i}^{\prime 2}+\left(1-b_{i j}\right) v_{\perp, i}^{\prime 2}+\sigma_{i j} v_{\phi, i j}^{\prime 2} \geq 0
$$

Particle trajectories satisfying inequality (3) are called passing trajectories. Particles which do not satisfy this condition are reflected back toward their initial location, implying that when condition (3) is invalid

$$
f\left(x_{i}, v_{\|, i}^{\prime}, v_{\perp, i}^{\prime}\right)=f\left(x_{i},-v_{\|, i}^{\prime}, v_{\perp, i}^{\prime}\right)
$$

This symmetry was used under identical hypotheses by Leroy and Mangeney [1984] and (without electric field) $W u$ [1984] to explain the formation of a field-aligned energetic electron beam upstream of nearly perpendicular shocks. The limit between reflected and transmitted particles hence is defined by an ellipsoid in velocity space if $B_{i}>B_{j}$ and $\phi_{i}>\phi_{j}$, and an hyperboloid (of one sheet) when $B_{i}<B_{j}$ and $\phi_{i}<\phi_{j}$ (other cases not being relevant to fast-mode shocks). We shall refer to the distribution function constructed from equation (2) as the Liouville-mapped distribution $f^{\mathcal{L}}$, noting that it is only defined for velocities satisfying (3).

[8] Overshoots are part of the quasi-stationary structure of supercritical shocks, and invalidate the monoticity hy- pothesis used in the previous paragraph [Gedalin and Griv, 1999]. Therefore when an overshoot is present in between the two locations to be mapped, we split the process by determining which particles can be mapped to the point where the fields reach their maximum value $B_{m}$ and $\phi_{m}$, assuming that these maxima occur at the same location, and then to the final location. In such a case, in addition to inequality (3) the passing particles velocities must also satisfy

$$
v_{\|, i}^{\prime 2}+\left(1-b_{i m}\right) v_{\perp, i}^{\prime 2}+\sigma_{i m} v_{\phi, i m}^{\prime 2} \geq 0
$$

Inequalities (3) and (5) generalize equations (7) and (8) of Gedalin and Griv [1999] for arbitrary $B$ and $\phi$ profiles as long as both fields reach their maxima at the same point. Hence overshoots decrease the number of passing trajectories, since they increase the number of mirror-reflected upstream particles and introduce mirror-reflection of downstream particles in addition to their possible reflection by the electric potential. Figure 1 shows that a strong enough overshoot can even imply that no downstream particle with nearly perpendicular pitch angle has a passing trajectory, in which case the mapping provides in particular very little information about the downstream perpendicular temperature. Furthermore, overshoots create effective potential wells for electrons with small perpendicular velocity where they can be trapped [Hull et al., 2001].

[9] Let us note that the hypothesis that the de HoffmannTeller potential and magnetic intensity reach their maxima simultaneously is quite reasonable given that the magnetic field, density, and electron temperatures are generally found to follow similar profiles. Furthermore, this hypothesis is approximately satisfied by potential profiles calculated from electron moments [Scudder et al., 1986a; Hull et al., 2000]. A potential reaching its maximum before the magnetic field would give more parallel momentum to incoming electrons and therefore increase the number of passing electrons, 
while the converse would be true in the opposite case. This, at least qualitatively, would not make a large difference.

[10] The mapping defined in equation (2) requires a yet unknown parameter, the de Hoffmann-Teller electric potential, while all the other parameters are easily determined by the observations. We choose the potential value which allows the best fit of the mapped distribution to the observed one. This forms the basis of the technique described in the next section.

\subsection{Estimation of the de Hoffmann-Teller Potential From Electron Distributions}

[11] Since the Liouville-mapped distribution is a functional of the cross-shock potential, the mapping can be used to find the electric potential difference which allows a "best fit" of the mapped distribution with the observed one. Hull et al. [2000] estimated in this way the potential difference across the shock, while the idea was used in a simpler form by Schwartz et al. [1988]. By a repeated fit of a mapped upstream distribution to all available electron distribution measurements during the shock crossing, one can get not only the cross-shock potential but its profile through the shock, as was suggested by Schwartz et al. [2000].

[12] The mapping described in the previous paragraph is applied using the measured magnetic field and the potential difference $\Delta \phi^{\prime}$ between the upstream and current satellite location, which appears in this procedure as a free parameter. This potential difference is then determined as the one which minimizes

$$
\xi\left(\Delta \phi^{\prime}\right)=\frac{1}{N-1} \sum_{i, j: f_{i j}^{\mathcal{L}}>0}\left|1-\frac{f^{\mathcal{L}}\left(x, v_{\| i}, v_{\perp j}\right)}{f\left(x, v_{\| i}, v_{\perp j}\right)}\right|,
$$

where $f$ is the observed phase space density at a given location, $f^{\mathcal{L}}$ the Liouville-mapped distribution at the same location and $N$ the number of terms in the sum. The sum in this equation is restricted to velocities of particles which have trajectories connecting the two locations and hence actually experience the potential difference. The fitting function $\xi$ was chosen to treat equally all values of the distribution function and is less sensitive to outliers than a more usual $\chi^{2}$. This form is chosen in particular to reduce the influence of values on the border of the exclusion region where the mapped phase space density is generally found to exceed the observed one [Scudder et al., 1986b]. Nevertheless, values of the phase space density below the fivecount level were rejected to avoid phase space density values with too large uncertainties. Choosing other similar expressions for $\xi$ does not modify appreciably the results.

[13] This procedure provides the potential in the de Hoffmann-Teller frame, which in particular has the advantage of being independent of coordinates along the shock surface, unlike for example the normal incidence frame potential [e.g., Scudder, 1995].

\section{Data Description}

[14] The three shocks studied in this paper are supercritical quasi-perpendicular bow shocks selected from periods of relatively steady upstream conditions. They were crossed by Cluster during data burst modes, when three-dimensional
(3-D) electron velocity distributions were available every satellite spin period (4 s) from the Plasma Electron and Current Experiment (PEACE) instrument [Johnstone et al., 1997]. However, because of telemetry limitations only a reduced form of the full 3-D measurements was transmitted to the ground. Electron data used in this study came from PEACE's Low Energy Electrostatic Analyzer (LEEA) and consist of 26 energy levels ranging from approximately $7 \mathrm{eV}$ to $1.7 \mathrm{keV}, 6$ polar and 32 azimuthal angular bins (3DX1 mode). High-temporal resolution FGM magnetic field data [Balogh et al., 2001] is interpolated at the times of individual measurement steps in order to compute pitch angles. Electron energy levels are corrected for the energy gained from the spacecraft floating potential using the spinaveraged EFW probe potential relative to spacecraft [Gustafsson et al., 1997], thereby removing the photoelectrons which contaminate the measurements. The velocity moments were computed by numerical integration using spacecraft potential-corrected distributions, and the electron number density was cross-checked using the plasma frequency provided by the WHISPER instrument [Décréau et al., 2001]. CIS/HIA [Rème et al., 2001] proton velocity moments used to estimate shock parameters were drawn from Cluster mission's Prime Parameters database. On all crossings the Cluster quartet was in a nearly equilateral tetrahedron configuration, with separation around $200 \mathrm{~km}$ for the 19 December 2003 and 23 January 2004 crossings and $1300 \mathrm{~km}$ for the 19 March 2005 crossing.

\section{Overview of the Shock Crossings}

[15] The magnetic field profiles of the shocks (Figure 2) are typical of supercritical quasi-perpendicular shocks, displaying a foot and a ramp, followed by an overshootundershoot cycle. Furthermore, they appear quite similar on the four spacecraft (using 4-s averages) for the 19 December 2003 and 23 January 2004 shocks, suggesting a rather stationary shock structure at the separation scale. However, for the 19 March 2005 shock, the shock is crossed by Cluster-4 (C4) nearly 3 min after $\mathrm{C} 1$ and $\mathrm{C} 2$ and displays a less steep profile, suggesting that the shock has slowed down during that interval (Figure 1, bottom).

[16] We have estimated the shock normals using the relative timings of ramp crossings between the four spacecrafts [Dunlop and Woodward, 2000; Schwartz, 2000] and using the Abraham-Shrauner coplanarity by calculating the unit vector along the direction of $(\Delta \mathbf{B} \times \Delta \mathbf{V}) \times \Delta \mathbf{B}$, where $\Delta$ indicates differences between the asymptotic downstream and upstream sides. These shock normals are also compared to those from an empirical model fit to large number of crossings [Peredo et al., 1995] and rescaled to fit the observed shock crossing location. The results are summarized in Table 1 and show good agreement between the methods. The shock normals estimated from the relative timings of the ramp crossings agree quite well with normals estimated on each satellite using the Abraham-Shrauner normal, suggesting the shock planarity at the satellite separation scale.

[17] The main shock parameters are shown in Table 2, where $(\mathrm{u})$ refers to the upstream (low-entropy) side of the shock and (d) to the downstream (high-entropy) side. The shocks have comparable strength (in terms of $B_{d} / B_{u}$ or $n_{d} /$ 

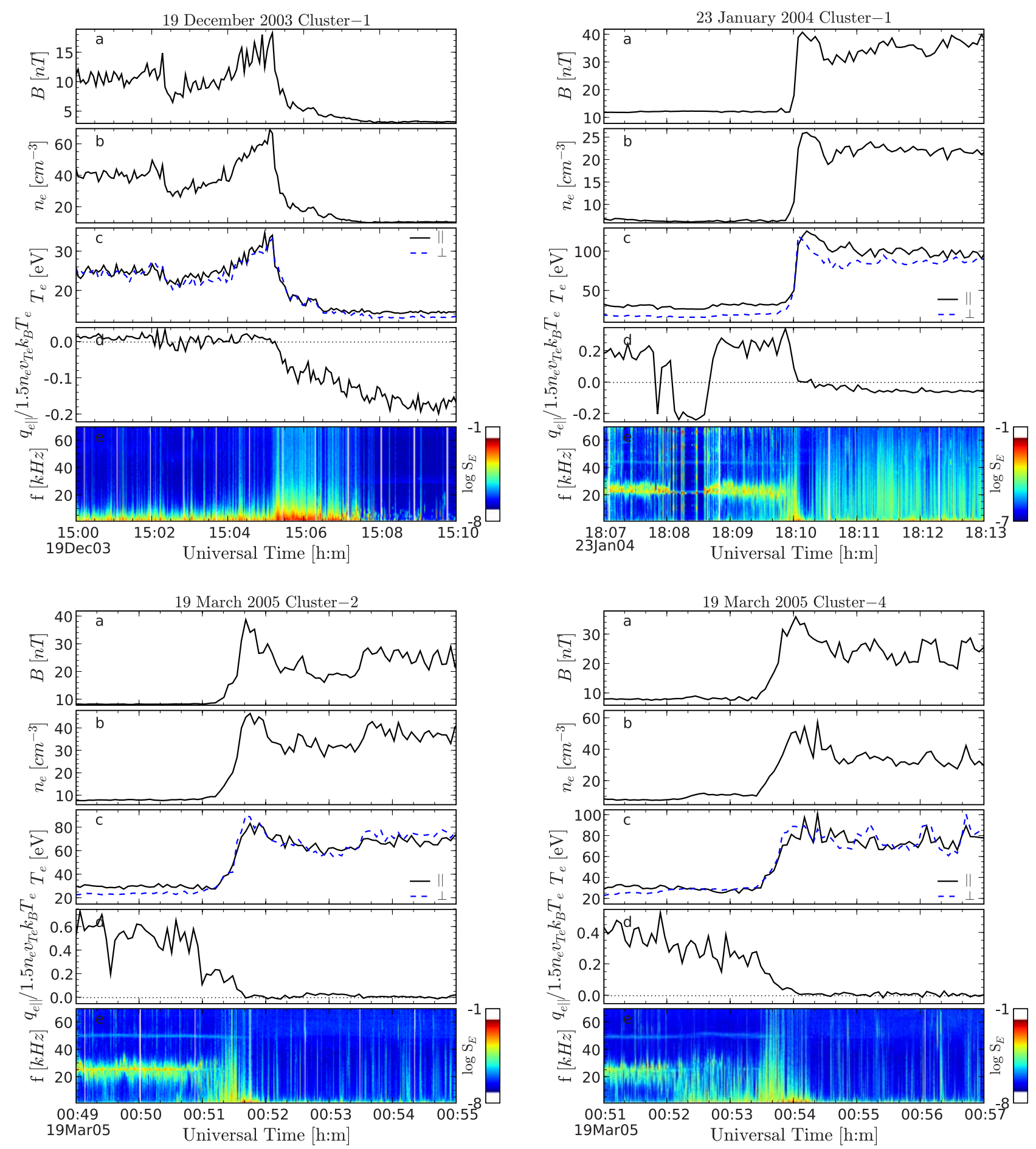

Figure 2. (a) Magnetic field amplitude, electron velocity moments (b) density, (c) parallel and perpendicular temperatures, (d) parallel heat flux normalized by $3 / 2 n_{e} v_{T e} k_{B} T_{e}$, and (e) electric field spectral density, on SC1 for the (top left) 19 December 2003 and (top right) 23 January 2004 and on (bottom left) SC2 and (bottom right) SC4 for the 19 March 2005.

$n_{u}$ ), propagation angles with respect to the upstream magnetic field around $72-81^{\circ}$ and Alfvénic Mach numbers from 5 to 10 . However, they display stronger differences in their plasma characteristics. For instance the ratios of electron kinetic to magnetic pressures $\beta_{e}$ range from 0.5 (23 January 2004) to 6 (19 December 2003). For this last shock, $\beta_{p}$ is high as well $\left(\beta_{p} \approx 3\right)$, and as usual in high- $\beta$ plasmas this shock has a particularly large overshoot. Highresolution magnetic field data shows its rather turbulent character, with possibly ripples propagating along the shock surface [Moullard et al., 2006]. Another important difference between these shocks is related to the dissipated solar 
Table 1. Comparison of Shock Normals From Different Methods: Timing, Abraham-Shrauner, and From a Shock Model [Peredo et al., 1995] Rescaled to Fit the Observed Shock Location ${ }^{\text {a }}$

\begin{tabular}{lcccc}
\hline Parameters & Units & 19 December 2003 & 23 January 2004 & 19 March 2005 \\
\hline $\mathbf{n}_{\text {timing }}(\mathrm{GSE})$ & - & $(0.79,0.61,-0.10)$ & $(0.90,0.40,0.18)$ & $(0.99,0.04,0.06)$ \\
$\mathbf{n}_{\text {AS }}$ GSE) & - & $(0.74,0.61,-0.27)$ & $(0.89,0.40,0.20)$ & $(0.99,-0.03,0.11)$ \\
$\mathbf{n}_{\text {model }}(\mathrm{GSE})$ & - & $(0.80,0.59,-0.11)$ & $(0.90,0.42,0.17)$ & $(0.98,-0.06,0.19)$ \\
$\mathbf{v}_{\text {shock }} \cdot \mathbf{n}_{\text {timing }}$ & $\mathrm{km} / \mathrm{s}$ & -4.5 & -7.5 & 8 \\
$\mathbf{v}_{\mathrm{HT}}(\mathrm{GSE})$ & $\mathrm{km} / \mathrm{s}$ & $(281,-392,-141)$ & $(501,-926,-474)$ & $(124,-3072,1254)$ \\
\hline
\end{tabular}

${ }^{\mathrm{a}}$ Also shown are shock velocity along normal and de Hoffmann-Teller frame velocity, both given in the satellite frame using timing results.

wind kinetic energy in the Normal Incidence (NI) frame. This parameter was shown in statistical surveys to be significantly better correlated with the electron heating than the shock geometry or Mach numbers [Thomsen et al., 1987; Schwartz et al., 1988; Hull et al., 2000], suggesting indeed that electron heating increases with the dissipated bulk kinetic energy. This is consistent with our estimations, since electron heating is most important for the shock with the largest difference in proton ram energy (23 January 2004) and weakest for the shock with the smallest one (19 December 2003).

[18] Electron heating is weaker at the 19 December 2003 shock than for the two others. For such relatively strong shocks, the electron pressure tensor tends to be more isotropic downstream than upstream (see parallel and perpendicular temperatures in Figure 2), although the downstream distributions are not necessarily strictly isotropic [Feldman et al., 1983]. The perpendicular temperature profile is slightly less smooth than the parallel one and tends to follow qualitatively the magnetic field profile, although $T_{e \perp} / B$ is not conserved in any of these shocks, which does not necessarily imply that the electrons' mag- netic moment is not conserved. Statistically, $T_{e \perp} / B$ was found by Schwartz et al. [1988] to increase more from upstream to downstream for higher electron thermal Mach number shocks, which is generally consistent with the results from Table 2, although there is a significant reduction of this quantity (almost by a factor 2) instead of an increase for the 19 December 2003 shock. The (parallel) heat flux vanishes near the magnetic field and electron temperature maximum and is elsewhere directed away from this point as could be expected from a thermodynamic point of view, overcoming upstream the solar wind heat flux but being negligible downstream when compared to the saturated heat flux $q_{s}=3 / 2 n_{e} v_{T e} k_{B} T_{e}$. It is worth keeping in mind, however, that due to the limited energy range of the instrument, these heat fluxes might not include the contribution from the electrons reflected and accelerated by the shock.

[19] Upstream distributions have some loss-cone-like features at low (thermal) energies, with a depression in the phase space density for electrons roughly moving along the magnetic field away from the shock (Figure 3), which are a sign of magnetic mirroring by the shock [Feldman et

Table 2. Summary of Main Shock Parameters at the Asymptotic Upstream and Downstream Locations ${ }^{\mathrm{a}}$

\begin{tabular}{|c|c|c|c|c|c|c|c|}
\hline \multirow[b]{2}{*}{ Parameters } & \multirow[b]{2}{*}{ Units } & \multicolumn{2}{|c|}{19 December 2003} & \multicolumn{2}{|c|}{23 January 2004} & \multicolumn{2}{|c|}{19 March 2005} \\
\hline & & (u) & (d) & (u) & (d) & $(\mathrm{u})$ & (d) \\
\hline$B$ & $\mathrm{nT}$ & 3.26 & 10.7 & 11.7 & 35.7 & 7.82 & 26.2 \\
\hline$n_{e}$ & $\mathrm{~cm}^{-3}$ & 11 & 37.8 & 6.59 & 21.9 & 7.76 & 19.3 \\
\hline$T_{e}$ & $\mathrm{eV}$ & 14.3 & 25.8 & 22.8 & 90.8 & 17.2 & 70.8 \\
\hline$T_{e \|} / T_{e \perp}$ & - & 1.08 & 1.01 & 1.64 & 1.1 & 1.14 & 0.934 \\
\hline$T_{e \perp} / B$ & $\mathrm{eV} / \mathrm{nT}$ & 4.3 & 2.40 & 1.60 & 2.46 & 2.10 & 2.76 \\
\hline$T_{p}$ & $\mathrm{eV}$ & 7.38 & 107.0 & 35.5 & 327 & 30.6 & 276 \\
\hline$\beta_{e}$ & - & 5.99 & 3.4 & 0.45 & 0.63 & 0.88 & 0.8 \\
\hline$\beta_{p}$ & - & 3.09 & 14.1 & 0.69 & 2.26 & 1.56 & 3.12 \\
\hline$v_{p}$ & $\mathrm{~km} / \mathrm{s}$ & 220 & 58.7 & 550 & 194 & 474 & 134 \\
\hline$\theta_{B n, \text { timing }}$ & $\operatorname{deg}$ & 75.9 & 86.4 & 72 & 79.2 & 81.2 & 89.2 \\
\hline$\theta_{B n, A S}$ & deg & 72.1 & 84.7 & 77.3 & 85.9 & 76.1 & 85.9 \\
\hline$\theta_{\text {Bn,model }}$ & $\operatorname{deg}$ & 70.3 & 81.4 & 75.6 & 84.7 & 72.6 & 82.9 \\
\hline $\begin{array}{l}\sigma_{B n, m o d e l} \\
\text { Proton ram energy, } m_{p} u_{p}^{2} / 2\end{array}$ & $\mathrm{eV}$ & 253 & 18 & 1580 & 196 & 1170 & 94.2 \\
\hline Alfvén Mach number, $M_{A}$ & - & 10.3 & 1.54 & 5.56 & 1.16 & 7.76 & 1.03 \\
\hline Fast Mach number, $M_{f}$ & - & 4.22 & 0.787 & 4.79 & 0.946 & 5.96 & 0.801 \\
\hline HTF electron thermal Mach number, $M_{T e}^{\prime}$ & - & 0.402 & 0.309 & 0.627 & 0.183 & 1.26 & 1.97 \\
\hline Electron plasma frequency, $f_{p e}$ & $\mathrm{kHz}$ & 29.8 & 55.2 & 23.1 & 42 & 25 & 39.4 \\
\hline Electron gyrofrequency, $f_{\mathrm{ge}}$ & $\mathrm{Hz}$ & 91.3 & 301 & 326 & 1000 & 219 & 734 \\
\hline Proton gyrofrequency, $f_{g p}$ & $\mathrm{~Hz}$ & 0.0495 & 0.163 & 0.177 & 0.543 & 0.119 & 0.398 \\
\hline Electron inertial length, $c / \omega_{p e}$ & $\mathrm{~km}$ & 1.6 & 0.864 & 2.07 & 1.14 & 1.91 & 1.21 \\
\hline Thermal electron gyroradius, $\rho_{e}$ & $\mathrm{~km}$ & 2.76 & 1.12 & 0.976 & 0.634 & 1.26 & 0.764 \\
\hline Proton inertial length, $c / \omega_{p p}$ & $\mathrm{~km}$ & 68.4 & 37 & 88.5 & 48.6 & 81.6 & 51.8 \\
\hline Thermal proton gyroradius, $\rho_{p}$ & $\mathrm{~km}$ & 85 & 98.2 & 52.2 & 51.7 & 72.2 & 64.6 \\
\hline Convected proton gyroradius, $v_{p 1} / \Omega_{c p}$ & $\mathrm{~km}$ & 707 & 215 & 494 & 161 & 636 & 190 \\
\hline
\end{tabular}

${ }^{\mathrm{a}}$ Frame-dependent quantities are given in the Normal Incidence frame (the shock rest frame where the upstream solar wind velocity is directed along the shock normal), unless a prime denotes the de Hoffmann-Teller frame (shock rest frame where bulk velocities are asymptotically field-aligned). Here (u) and (d) refer to upstream and downstream (low and high entropy sides of the shock), respectively. 

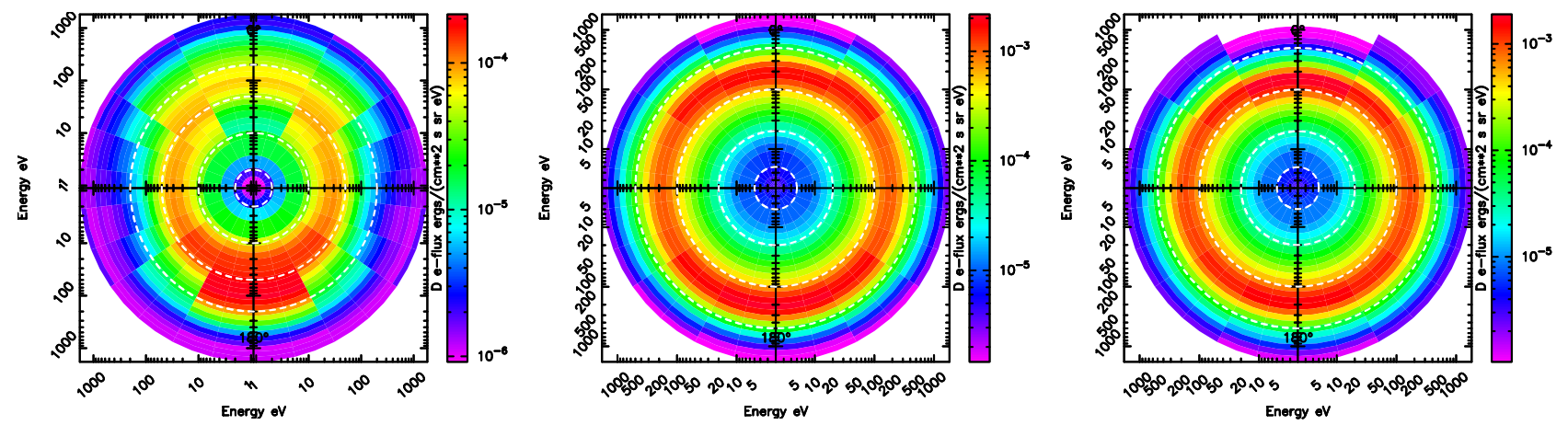

Figure 3. Differential electron energy flux on C1 for the 23 January 2004 shock, upstream (1809:11, left), slightly behind the ramp (1810:16, middle), and downstream (1811:28, right). The horizontal axis corresponds to the perpendicular (gyration) speed while the vertical axis corresponds to the parallel component. The figures have been mirrored around $v_{\perp}=0$ for readability.

al., 1983; Scudder, 1995; Lobzin et al., 2005]. These features are more noticeable for the stronger 23 January 2004 and 19 March 2005 shocks. Behind the ramp, in the overshoot, the electron distribution have the flat-top shape typical of strong shocks on top of which sit a weak parallel sheath-directed "beam" (Figure 4, middle) [Feldman et al., 1983], although both features are very weak for the 19 December 2003 shock. Figure 4 (right) also shows that downstream of the shock the beam is not always sheathdirected but sometimes on the contrary appears to be shockdirected even though no significant rotation of the magnetic field was found, which is not consistent with the local acceleration of electrons by the cross-shock potential but rather electrons travelling in the magnetosheath from another part of the shock [e.g., Feldman et al., 1983]. The 23 January 2004 shock has the strongest electron temperature anisotropy both upstream and downstream (Table 2), while the upstream parallel velocity distribution is strongly nonMaxwellian (Figure 4, left). This shock also displays the strongest beam near the downstream directed shoulder of the velocity distribution. To the 19 December 2003 shock correspond very faint upstream plasma emissions, as shown by the electric field spectra measured by the Whisper instrument (Figure 2, top left), suggesting a very small density of upstream-reflected electrons and/or their spread near thermal energies. The Langmuir waves are much more intense upstream of the 23 January 2004 shock (Figure 2, top right), apart from a brief interval when the heat flux carried by particles coming from the shock vanishes, which probably corresponds to a short excursion of the satellite outside of the electron foreshock. Nevertheless, no beam of reflected electrons could be clearly identified in the data. Plasma frequency oscillations decrease when entering the foot, while the intensity of electric fluctuations at lower frequencies increases as is usually the case in the ramp.

\section{Liouville Mapping Application to Cluster/ PEACE Data}

\subsection{Comparison of Mapped and Observed Velocity Distributions}

[20] The mapping technique described in section 2 (including the effect of the overshoots), is applied here to PEACE data, which is first transformed to the de HoffmannTeller frame, mapped to another location, and transformed back to the satellite frame for comparison with measure-
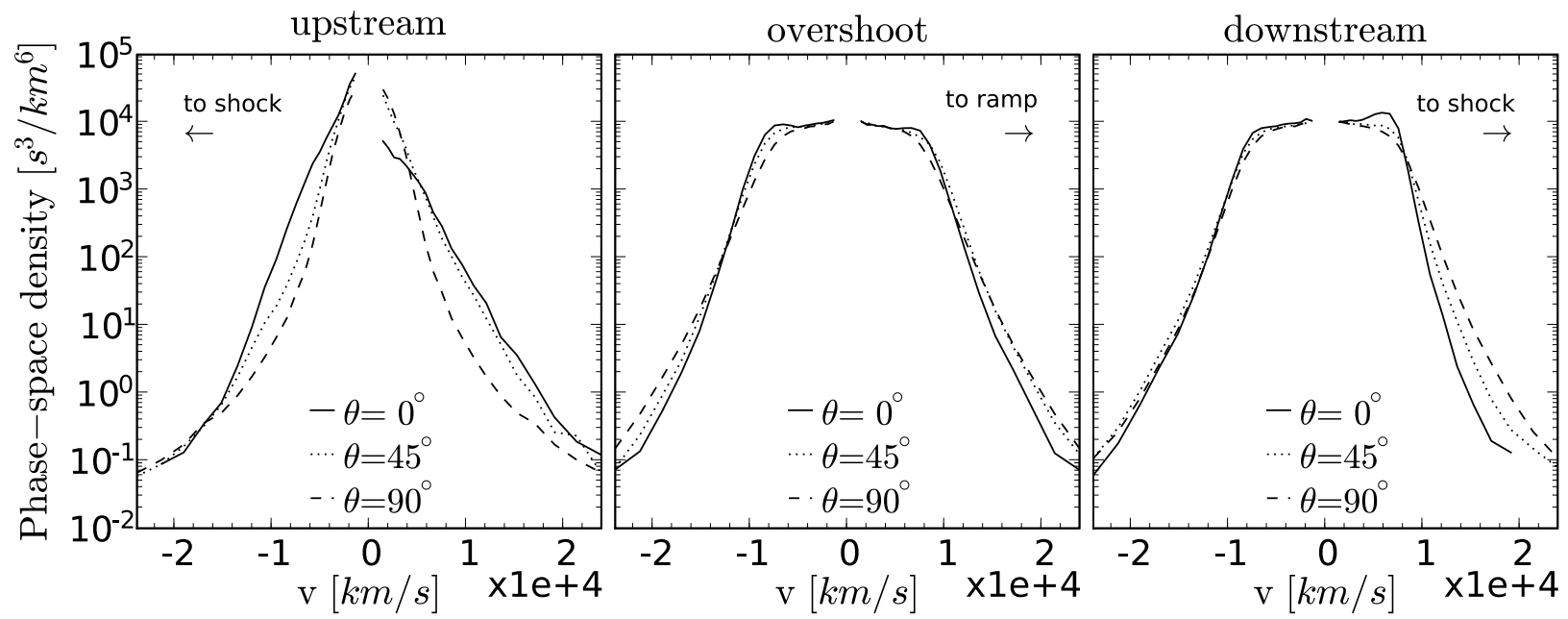

$\mathrm{C} 1$

Figure 4. Cuts of the electron velocity distribution on C1 for the 23 January 2004 shock, upstream, in the overshoot and downstream (same times as Figure 3). 

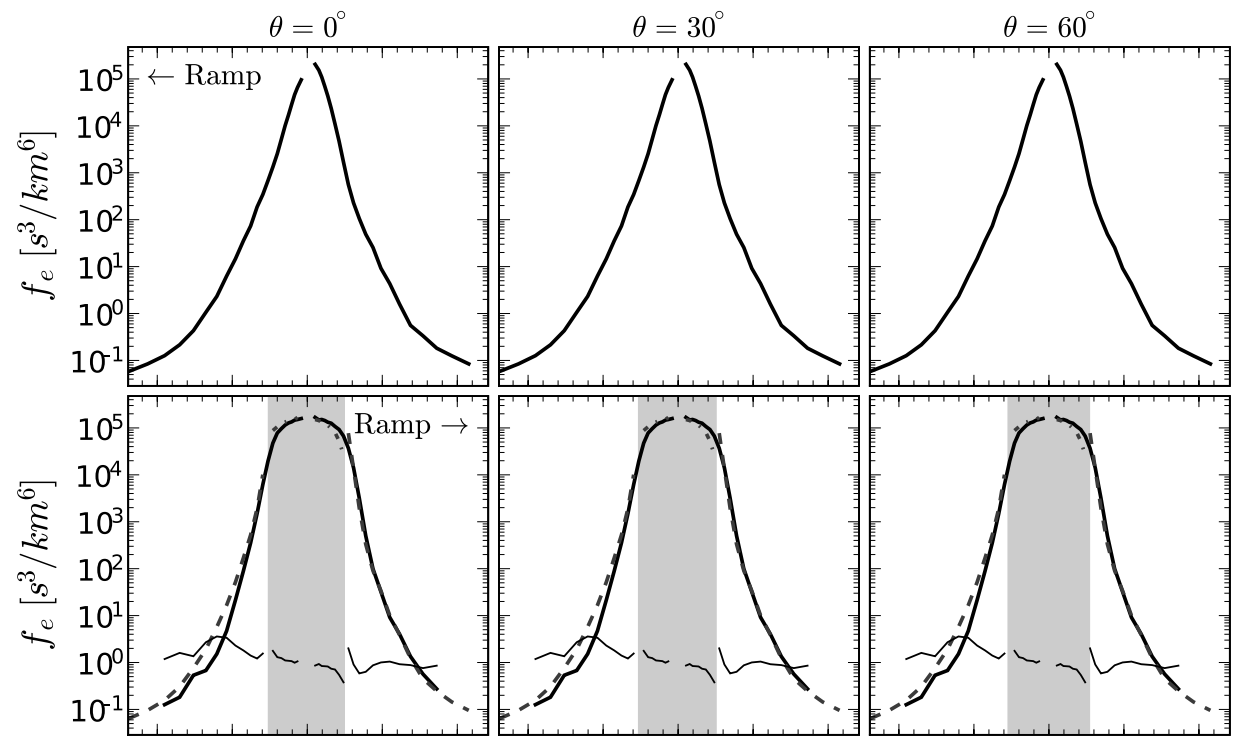

C1 15:10:00
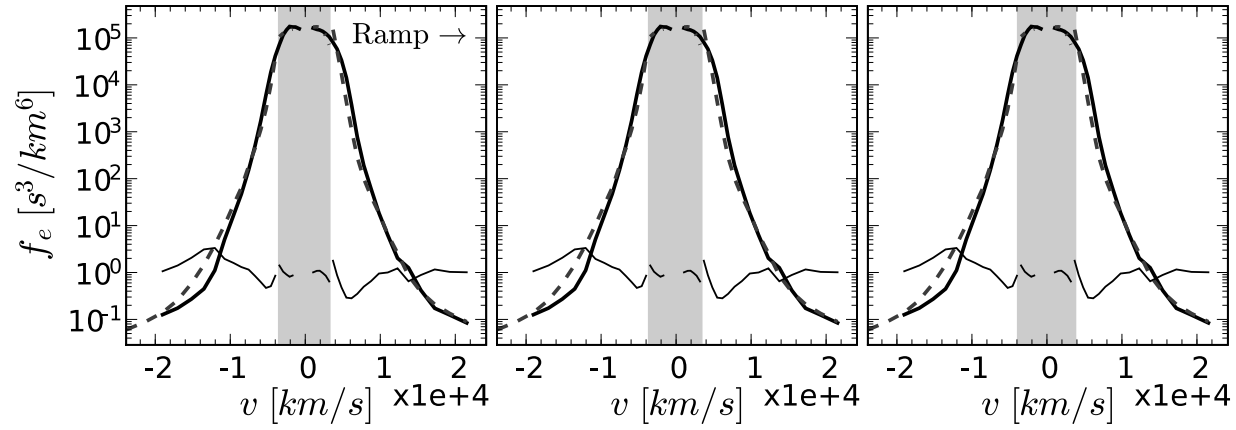

Downstream

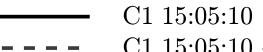

-..... C1 15:05:10 つ

Ratio

Figure 5. Cuts at 0,30 , and $60^{\circ}$ pitch angles (in the satellite frame) of the measured velocity distribution (thick continuous line), distribution mapped from upstream (dashed) and distribution of downstream-reflected particles (dash-dotted) for the 19 December 2003 shock on $\mathrm{C} 1$. The original upstream distribution being mapped to the other side of the shock is shown at the top, and comparisons are shown slightly beyond the ramp in the overshoot (middle) and further downstream (bottom). The shaded areas correspond to regions of nonpassing particles also shown on Figure 1.

ments. Comparison between cuts at different pitch angles of the downstream electron velocity distribution with the one mapped (using equation (2)) from upstream to locations slightly beyond the ramp and further downstream are shown in Figures 5 and 6. The figures show also the downstream distribution which was flipped around $v^{\prime}=0$ in the de Hoffmann-Teller frame to test the symmetry defined by equation (4) for the part of velocity-distribution which do not satisfy the conditions (3) and (5) (and therefore correspond to reflected particles). The upstream and downstream states for the mapping were chosen closer to the shock than the "asymptotic" locations, but far enough not to be affected by the shock macroscopic fields. For the 19 December 2003 and 23 January 2004 shocks, the upstream and downstream distributions were taken from the same satellite at different times. On 19 March 2005, C2 and C4 were roughly aligned with the upstream and downstream magnetic fields $\left(50^{\circ}\right.$ upstream and $41^{\circ}$ downstream) and were distant enough to allow mapping of the upstream distribution on $\mathrm{C} 4$ to the simultaneous downstream one on $\mathrm{C} 2$. Using simultaneous data from a pair of approximately magnetically connected satellites ensures a better connection of the two locations by the electron trajectories and therefore relaxes a bit the strictness of the shock stationarity and planarity hypothesis. To our knowledge, the only published comparisons of mapped and observed distributions for nonzero pitch angles were carried out by Hull et al. [1998; see also Hull et al., 2000] for a very weak shock, and by Fitzenreiter et al. [2003] at an interplanetary shock, although in this case the analysis is hampered by the high velocity of the shock in the spacecraft frame. Both have found reasonable agreements between the mapped and observed distributions.

[21] The cuts on Figures 5 and 6 show that the mapped distributions correspond approximately to the observed one for all pitch angles, with even remarkable agreement for the shock with weakest electron heating (19 December 2003). However some discrepancies occur, and these differences are stronger for shocks with stronger heating as shown on Figure 6 for the 19 March 2005 shock. The mapped distribution appears steeper than the observed one, which results in an excess of the mapped distribution with respect to the observed one around the parallel direction at the lowest energies (middle and bottom left of Figure 6). This was already noticed by Scudder et al. [1986b] on parallel cuts of the distributions. Conversely, at larger pitch angles 


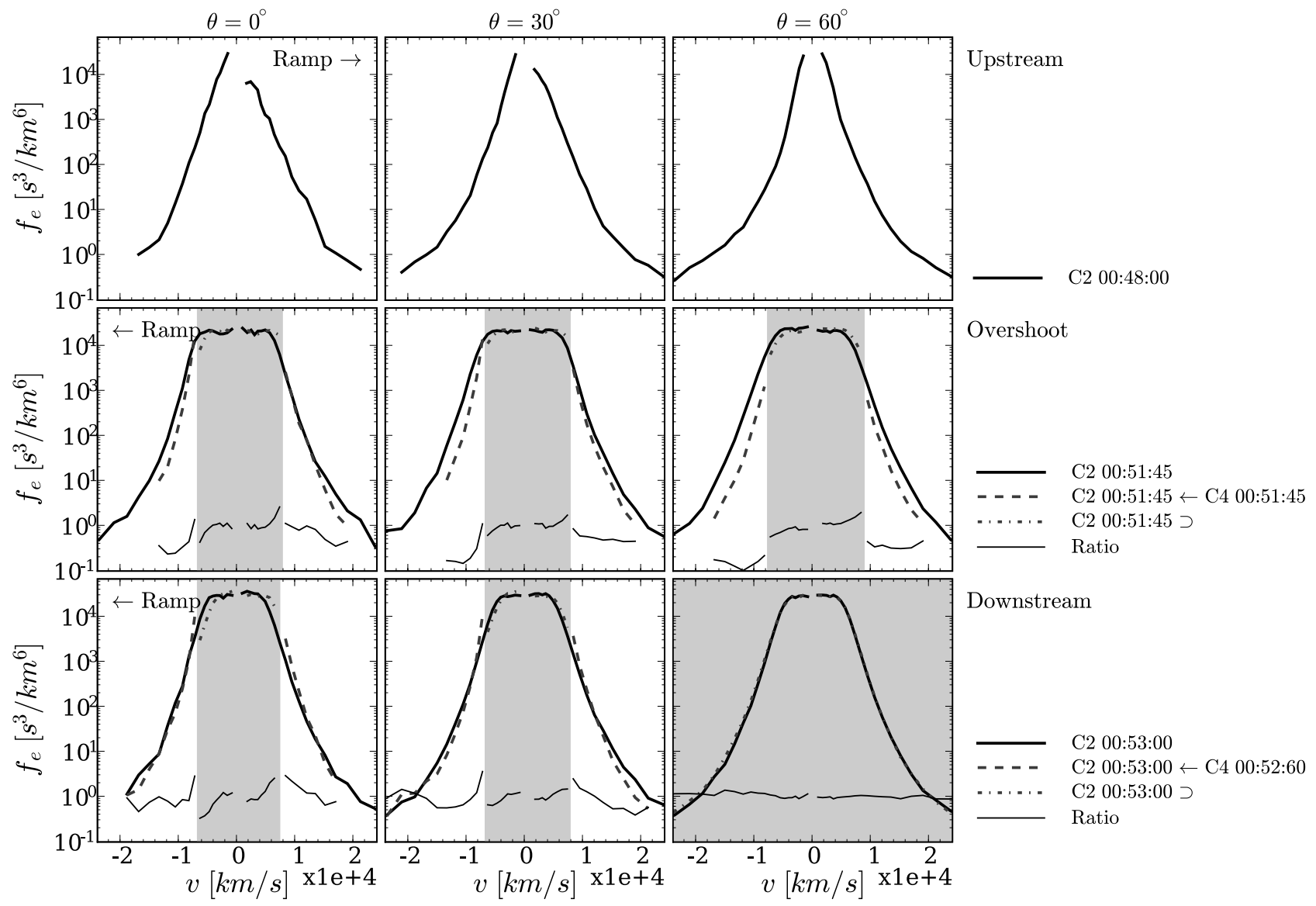

Figure 6. Same as Figure 5 for the 19 March 2005 shock. For this shock, the observed distributions on $\mathrm{C} 2$ are compared to mapped ones coming from simultaneous measurements on $\mathrm{C} 4$, while $\mathrm{C} 4$ remains in an upstream location roughly connected to $\mathrm{C} 2$ by magnetic field lines.

the mapped distribution is found to significantly underestimate the observed one (middle right of Figure 6). Some lack of symmetry around $v^{\prime}=0$ in the reflected part of the downstream distribution (equation (4)) is on the contrary more noticeable for the 19 December 2003 (bottom left of Figure 5), as shown by the discrepancy between the reflected (dotted curve) and observed distribution. This may indicate the presence of irreversible effects acting on low energy particles downstream, although one has to keep in mind the possible experimental source of errors such as possibly inaccurate determination of the de HoffmannTeller velocity. Finally, there is always a discontinuity in the velocity distribution predicted by the mapping at energies on the border between reflection and transmission. Such discontinuities are not expected physically, as they should be smoothed by other processes.

\subsection{Electron Guiding-Center Dynamics}

[22] As described in section 2, an estimate of the electric potential profile can be derived from the mapping by fitting the mapped distribution to the observed one. This estimate will be compared to others in section 6 . Here we use it to find in a self-consistent manner the guiding-center trajectories of electrons which would conserve their magnetic moment and energy in the HT frame. These guiding centers move in an effective potential $\hat{U}^{\prime}=\mu B-e \phi^{\prime}$ (equation (1)) quite analogous to the electric potential of a double layer, and their trajectories can be classified as passing, reflected or trapped. The shape and size of $\hat{U}^{\prime}$ varies widely for different values of $\mu$, from mostly field-aligned particles $(\mu \approx 0)$ for which the effective potential is essentially the electric potential, to those with large $\mu$ which are mostly sensitive to the magnetic field.

[23] Figures 7 and 8 show the effective potential $\hat{U}$ and the trajectories plotted as isocontours of the energy (equation (1)) transformed back into the satellite frame, and superimposed onto the distribution function $f\left(x, v_{\|}, \mu\right)$ measured in the satellite frame for different values of $\mu$. In the drift approximation, these trajectories should correspond to the contours of the phase space density. This appears from the figures to be globally well verified, for all types of trajectories. A similar analysis restricted to $\mu=0$ was performed by Scudder et al. [1986b] for a high $\beta$ quasiperpendicular shock, also showing good overall agreement. Here we show results for $\mu>0$, which as $\mu$ increases show more and more trajectories reflected on either side of the shock, or trapped trajectories in the effective potential wells. Passing trajectories appear to follow in general quite well the contours of the distribution function, with increasing accuracy for larger parallel velocities. At lower energies, and particularly for reflected particles one notes however that for the reflected particles (both upstream and downstream), the symmetry of $f$ around $v_{\|}=0$ (equation (4)) is 

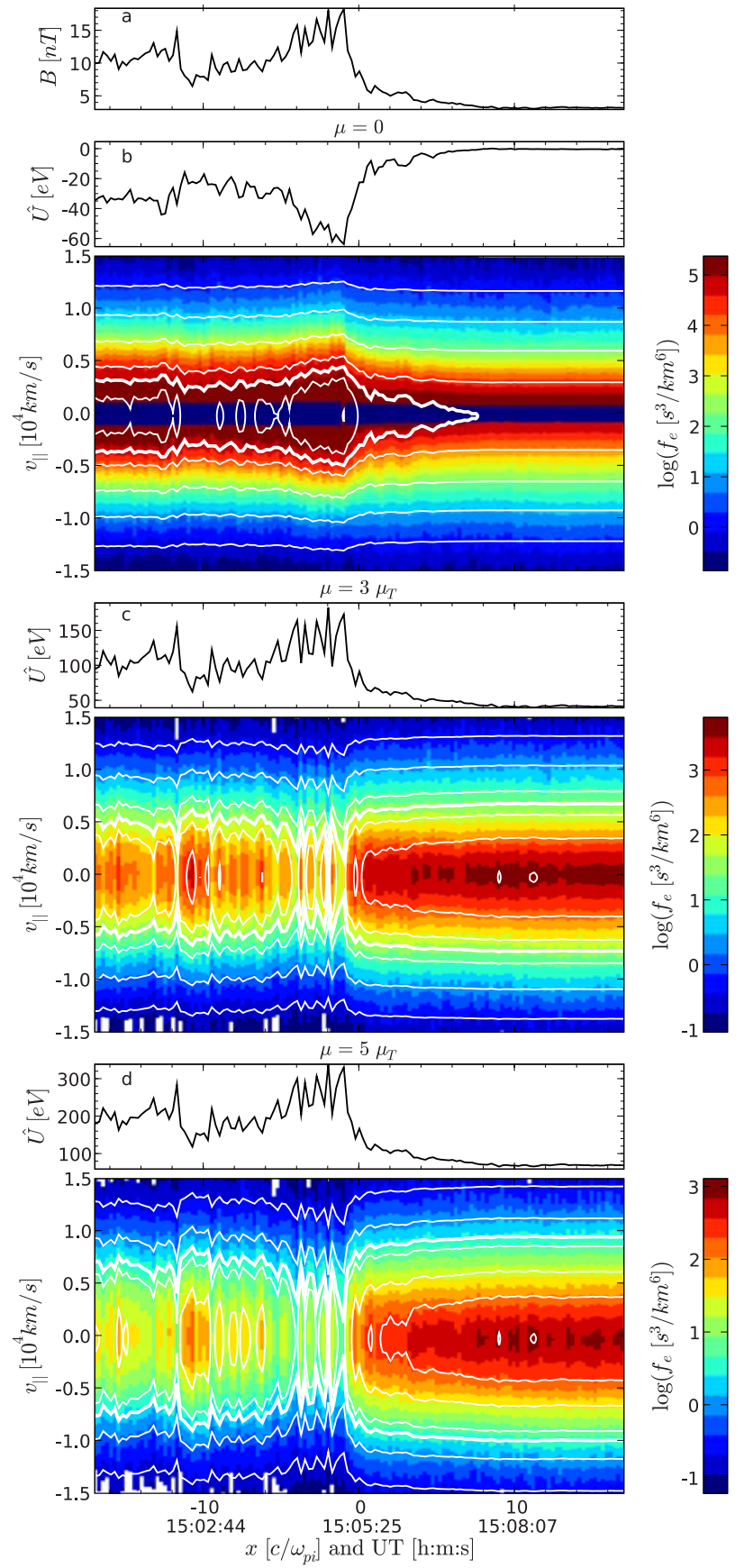

Figure 7. Phase-space density as a function of time and parallel velocity for different fixed values of the adiabatic invariant for the 19 December 2003 shock. In each subplot, the top shows the effective potential (equation (1)) calculated from the measured magnetic field and the electric potential inferred from the mapping. The bottom shows the measured velocity distribution $f\left(v_{\|}, \mu\right)$ for given (constant) $\mu$. White curves on the bottom correspond to constantenergy curves (guiding-center trajectories) for the energy defined by equation (1). The thicker curve corresponds to the separatrix between passing/nonpassing trajectories. sometimes broken. This is particularly visible on the upstream parts of Figures $8 \mathrm{c}$ and $8 \mathrm{~d}$, confirming findings from section 5. These electrons around the separatrix between reflection and transmission are those which spend the longest time in the shock layer, allowing them to be more sensitive to extra effects not included in the mapping. It is also worth noting that this violation of symmetry also occurs for trajectories which do not enter the ramp, suggesting that irreversible effects are not necessarily confined to the ramp. Furthermore, plots for higher adiabatic invariant clearly show for the first time in experimental data trapped trajectories along which the

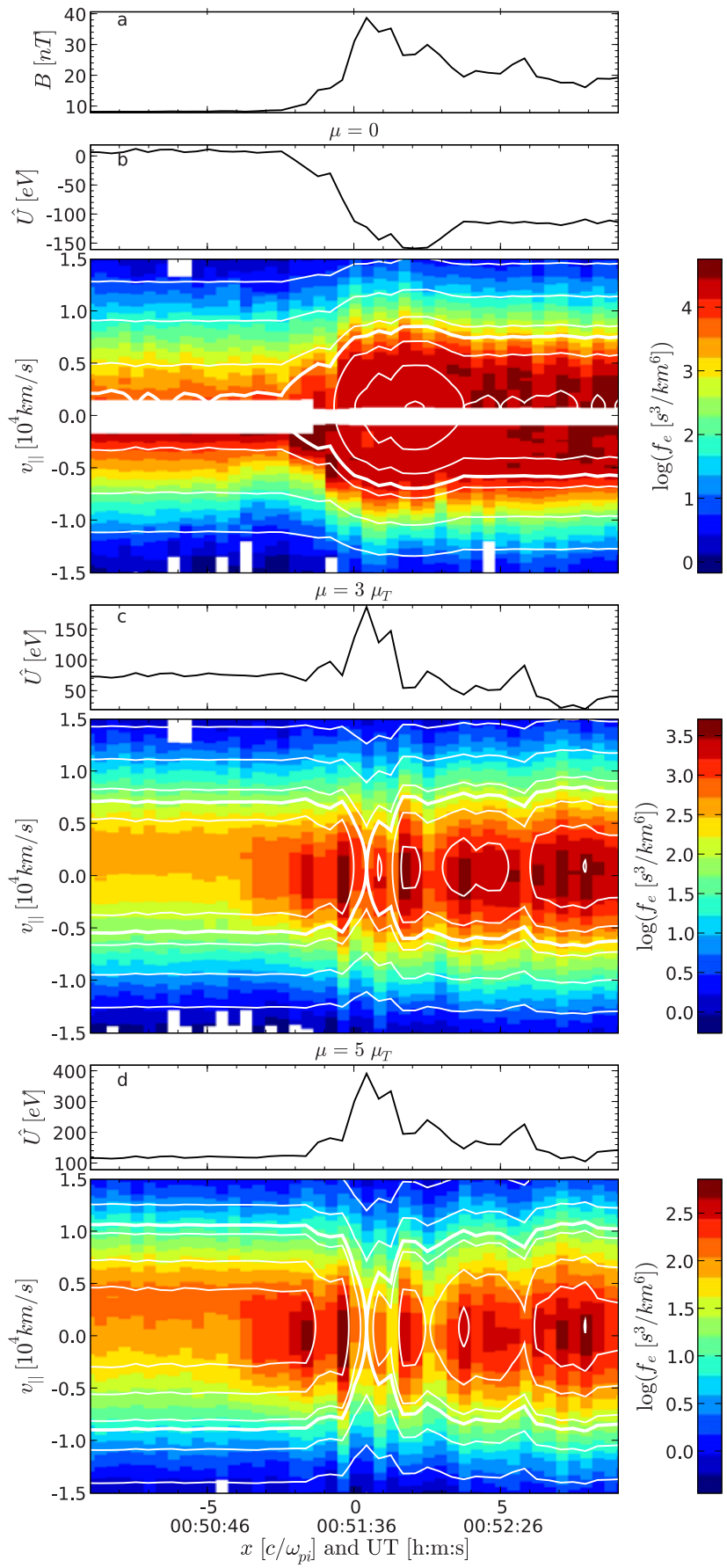

Figure 8. Same as Figure 7 for the 19 March 2005 shock. 

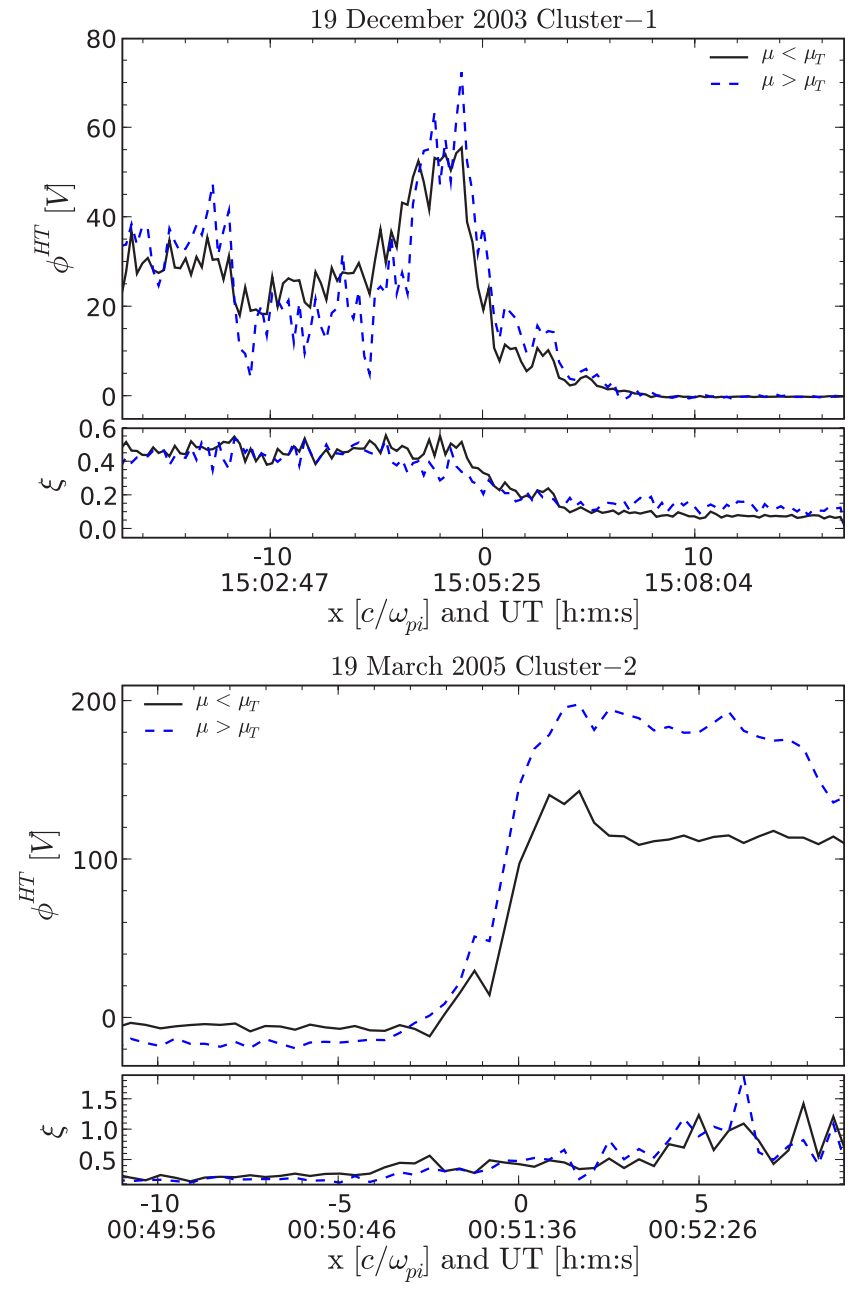

Figure 9. Cross-shock potential estimation using electrons within a restricted range of adiabatic invariants, for the (top) 19 December 2003 and (bottom) 19 March 2005 shocks. The solid lines correspond to $\mu<T_{e \perp}, u / B_{u}$ and the dashed ones to $\mu>T_{e \perp, u} / B_{u}$. The lower panels show values of the fitting function (equation (6)).

phase space density is conserved. These trapped orbits are found for nearly field-aligned particles in the undershoot region, and downstream of the shock for particles with large $\mu$, particularly in the downstream overshoot-undershoot cycle. For particles to fill these trapped orbits additional effects must be considered [Hull et al., 2001], such as largescale and slow (compared to trapped particle bounce time) temporal variations of these structures or pitch-angle scattering. Finally, one observes sharp and nonmonotonic variations of the effective potential $\hat{U}$ which reflect the nonstrict proportionality of $\phi^{\prime}$ and $B$.

\subsection{Electric Potentials From Different Subsets of Electrons}

[24] Since we have found that the mapping does not apply equally well to particles of all energies, it can be interesting to check if electrons with different adiabatic invariants would provide different estimates of the cross-shock potential. This can be done by restricting the sum in equation (6) to electrons in a given range of magnetic moment $\mu$. In order to keep a sufficient number of data points for the fit, only two ranges of $\mu$ where considered, below and above the $\mu_{T}=T_{e \perp} u / B_{u}$.

[25] Figure 9 shows the electric potential profiles evaluated in this way for the 19 December 2003 and 19 March 2005 shocks. The figures shows as well the values of the fitting function $\zeta$ which provide some information (however limited) on the quality of the fit, which are found to be comparable for the potential estimates. If the hypotheses behind the mapping applied equally well to electrons of all energies, there would be no significant difference between the two curves. For the 19 December shock, the difference between the potential estimates indeed remains small. Nevertheless the high- $\mu$ potential slightly increases faster than the low- $\mu$ one in the foot and the ramp. These differences are considerably amplified for the 19 March shock, resulting in a significant discrepancy downstream. The low$\mu$ potential appears to be the one closest to the potential estimated using the full energy range.

[26] This is consistent with the previously found fact that for stronger shocks the mapping tends to overestimate the observed distribution in the parallel direction (at least, at low energies) and underestimate it in the perpendicular direction. This also tells that additional effects neglected in the mapping and responsible for these differences affect differently electrons with large and small magnetic moments.

\subsection{Higher Time Resolution Electric Potential Profiles}

[27] The mapping approach also allows an estimation of the electric potential at a subspin temporal resolution. Indeed, the fitting function in equation (6) does not require a knowledge of the full velocity distribution. Therefore even if it takes a satellite spin to make a full 3-D measurement of the electron distribution function by a series of fast sweeps in energy/angle at nearly constant spin azimuth, as soon as the sum in equation (6) can be run over a sufficient number of data points one can get an estimate of the value of the potential. Such partial information from individual sweeps was already used by Montgomery et al. [1970] in an attempt to study electron heating through the shock ramp at a high temporal resolution.

[28] There are two clear limitations to this approach however. First, the instrument tends to sample particles with rather perpendicular (in the satellite frame) pitch angles unless the magnetic field is contained in its field of view. This is a problem since these particles often fall into the mapping's exclusion region (where equation (3) is not valid) and thus are useless to estimate the potential. Second, the fewer the data points are used to estimate the fitting function, the lower the accuracy of the determined electric potential. However we have found that using data from five sweeps, corresponding to a time resolution of $625 \mathrm{~ms}$, and keeping values for which the sum in equation (6) was calculated over at least 10 velocities, provide an electric potential profile at significantly higher time resolution and in good agreement with the spin-frequency one (Figure 10 for the 19 December 2003 shock). It nevertheless displays oscillations at the half-spin frequency, particularly notable in the solar wind, related to the magnetic field coming in and out of the instrument's field of view, and the amplitude of these oscillations provide a natural estimation of the error 


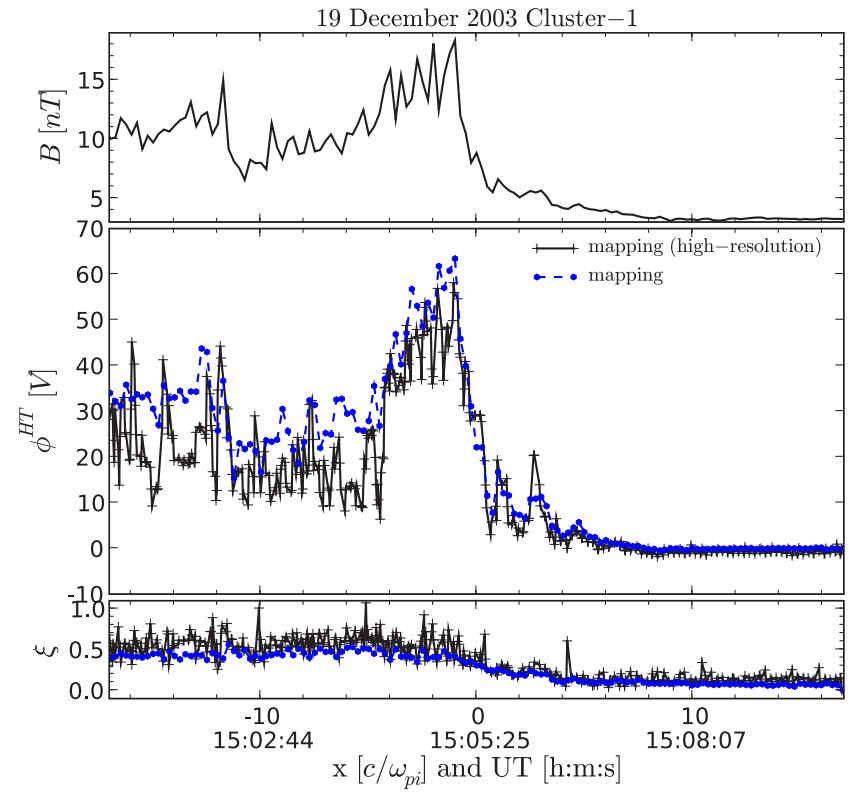

Figure 10. Higher temporal resolution (625 ms) HT electron potential from the mapping technique (dots), and comparison with the spin-resolution result (crosses) for the 19 December 2003 shock. Top panel shows high-resolution magnetic field subsampled at the same temporal resolution as the potential.

added by not using the full velocity distribution measurement. Nevertheless, the potential gradient tends to be slightly less uniform than suggested by the lower-resolution measurements, with corresponding HT electric fields reaching a few $(\simeq 5) \mathrm{mV} / \mathrm{m}$ from the high-resolution potential compared to $0.5 \mathrm{mV} / \mathrm{m}$ from spin-resolution potential (19 December 2003 shock).

[29] This result suggests that an important part of the potential increases across the shock occurs into the foot, followed by a sharp increase in the ramp. Similar behavior is found in numerical simulations [e.g., Scholer et al., 2003]. At this temporal resolution, the potential appears smoother and more monotonic than the magnetic field.

\section{Comparison of Cross-Shock Potential Profiles With Fluid Estimates}

\subsection{Estimation of the de Hoffmann-Teller Potential From Electron Velocity Moments}

[30] The de Hoffmann-Teller cross-shock potential derived in the previous section from the Liouville mapping can also be estimated from the electron fluid equations under the assumptions of shock planarity and stationarity, a technique used by Scudder et al. [1986a] and Hull et al. [2000].

[31] This approach relied on the hypotheses that electrons have a gyrotropic distribution with a bulk velocity in the de Hoffmann-Teller frame which is field-aligned through the layer and not only in the asymptotic upstream and downstream states (see Scudder [1987] for a justification). Furthermore, resistivity and viscosity are neglected. In such conditions, the steady state electron momentum equation integrated across a planar discontinuity in the de HoffmannTeller frame yields [Goodrich and Scudder, 1984]:

$$
e \Delta \phi^{\prime}=\int_{x_{1}}^{x_{2}} \frac{1}{n_{e}}\left[\frac{d P_{e \|}}{d x}-\left(P_{e \|}-P_{e \perp}\right) \frac{d \ln B}{d x}\right] d x
$$

which corresponds to the integration of the ambipolar electric field along the shock normal. This method has the advantage of requiring only estimates of the pressure tensor and density, and therefore does not necessitate any transformation to the HT frame. Under the same conditions, and neglecting any energy sink/source, the stationary electron energy equation can be integrated to:

$$
e \Delta \phi^{\prime}=\Delta\left[\frac{m}{2} u_{e \|}^{\prime 2}+\frac{3}{2} T_{e \|}+T_{e \perp}+\frac{q_{e \|}}{n_{e} u_{e \|}^{\prime}}\right] .
$$

This expresses the potential as a difference between terms evaluated at two different locations [Hull et al., 2000], which does not require knowledge of these parameters in between and does not pose problems of numerical integration of undersampled functions which may arise using equation (7). Furthermore, this expression has three contributions with clear physical interpretation. The first is due to the change in bulk flow energy of the electrons, $\Delta \frac{m}{2} u_{e \|}^{\prime 2}$, which is generally negligible. The middle terms would reduce in an isotropic plasma to the cross-shock potential difference if the electrons obeyed an adiabatic law $p \propto n^{\gamma}$, giving $\Delta \phi=\frac{\gamma}{\gamma-1} \Delta T$, with $\gamma=5 / 3$. The last term in equation (8) involves the heat flux and hence represents the nonadiabatic (in the thermodynamic sense) contribution to the potential. It as well depends on shock geometry, through the parallel de Hoffmann-Teller electron bulk velocity.

\subsection{Electric Potential Profiles}

[32] The electric potential estimates derived from electron moments are compared to the one from the Liouville mapping in Figure 11. The figure shows as well values of the fitting function $\xi$ defined in equation (6). As in the previous section, the mapping is done from one satellite to the same one at different times excepted for the 19 March 2005 shock when simulatenous data from two roughly fieldaligned satellites is used. For this last shock, the potential estimated from equation (8) also uses data from the same pair of satellites.

[33] The potential estimates remain satisfactorily constant in the solar wind, the one from the energy equation generally exhibiting the largest fluctuations. The three methods are in good agreement for the shock which exhibits the smallest heating (19 December 2003, shown on top). The potential difference is in order of magnitude comparable to the electron temperature difference, as can be expected for an ambipolar electric field. One also notes that the potentials from the momentum and energy fluid equations are in good agreement for each of the three shocks. Discrepancies between methods are more important for the stronger shocks. In particular, downstream the difference between the fluid and the mapping results is almost as large as the downstream thermal energy for the 23 January 2004 shock (Figure 11, middle). It is also the shock for which the fitting function $\xi$ reaches the largest values, indicating a less 

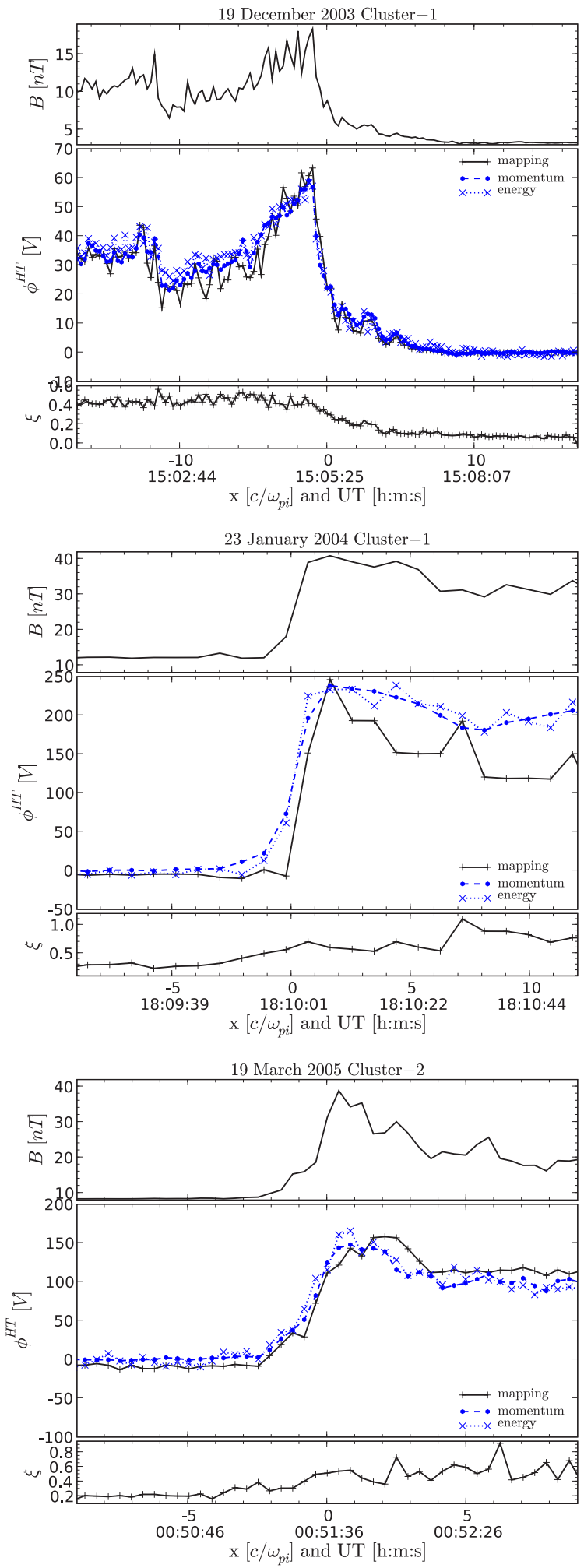

Figure 11. Magnetic field amplitude and cross-shock potential from the three methods, for the (top) 19 December 2003, (middle) 23 January 2004, and (bottom) 19 March 2005 shocks. Distances from the ramp along the shock normal are normalized with respect to the upstream ion inertial length $c / \omega_{p i}$. satisfying fit. Finally, let us note that for the 19 March 2005 shock, the potential resulting from mapping $\mathrm{C} 4$ data to $\mathrm{C} 2$ is very similar to the one obtained by mapping $\mathrm{C} 2$ to itself. Since the use of an appropriate satellite pair can only improve the results, this indicates that the planarity and stationarity hypotheses apply satisfactorily here.

[34] Globally, the potential profiles follow those of the magnetic fields, starting to increase in the foot and reaching a maximum in the region of the overshoot, meaning that they vary on comparable scales. A similar conclusion was reached using potential estimated from electron moments by Scudder et al. [1986a] and Hull et al. [2000], and numerical simulations show similar results [Scholer et al., 2003; Lembège et al., 2003]. One notes however that the scales are quite significantly different for the mapping electric potential on the 23 January 2004 (Figure 11, middle), with not much increase in the foot and sharp gradients in the ramp and therefore stronger electric fields. It must be noted however that this can be partly an artifact of time-aliasing since this crossing is quite short, but there can be more physical reasons involved.

[35] All three methods rely on the shock planarity and stationarity. They may also equally suffer from time aliasing in the ramp, since they rely on electron data which take a full satellite spin to collect (although as discussed in the previous section this can be improved for the mapping method). Nevertheless, other aspects of the methods differ and each has its limitations. For instance, the integral in equation (7) can be miscalculated due to insufficient temporal (and hence spatial) sampling for shorter crossings (23 January 2004 and 19 March 2005). Moreover, the integrand involves gradients which need to be approximated. The energy equation result in equation (8) does not suffer from such numerical integration problem, but involves a heat flux, a higher-order moment, for which the uncertainties are typically larger than for example the pressure tensor. Furthermore, equation (8) involves the electron bulk velocity in the de Hoffmann-Teller, which is hampered both by the difficulty of accurately determining the electron bulk velocity and the de Hoffmann-Teller frame velocity in the satellite frame (this being increasingly difficult when $\theta_{B n}$ gets closer to $90^{\circ}$ ). Finally, both methods neglect the perpendicular component of the velocity, whose contributions according to Scudder [1987] are in decreasing order of magnitude due to electron pressure anisotropy, inertia and bulk resistivity and are negligible for low de HoffmannTeller electron thermal Mach number $M_{T e}^{\prime}$ in fast-mode shocks. Although discrepenties between the fluid and mapping methods are here not the largest for the shock with the highest $M_{T e}^{\prime}$, they may nevertheless suggest that the electron pressure anisotropy (and possibly other effects) break the field-aligned approximation in this case. Equations (7) and (8) can be quite straightforwardly generalized to non fieldaligned flows, but this approach retains in particular the problems associated with the accurate determination of the electron HT bulk velocity.

[36] The hypotheses behind the mapping result were presented in section 2 and are less stringent than for the fluid result. They would be violated for instance if fluctuations within the shock provided significant energy/pitch-angle diffusion or if sharp gradients demagnetized the electrons (and this would also break hypotheses behind the fluid 
methods), as shall be discussed in the next section. However, when these hypotheses apply satisfactorily to thermal electrons, this technique has the advantage of relying only on electrons whose trajectory crosses the shock and hence which experience the full effect of the potential, whereas results from fluid theory rely on velocity moments which encompass all electrons. To the very least, this makes the potential estimated from the mapping more consistent with the electron dynamics discussed in this paper. Furthermore, it treats all these passing electrons equally, whereas, depending on their order, moments favor certain energy ranges. Nevertheless, the method remains sensitive to the HT-frame velocity, which is increasingly problematic for more perpendicular shocks.

\section{Discussion}

[37] Based upon the assumptions of shock planarity and stationarity, and conservation of the electron first adiabatic invariant and energy in the de Hoffmann-Teller frame, an estimate of the cross-shock electric potential was retrieved by fitting mapped velocity distributions to observed ones. A quite remarkable correspondence between the mapped and observed distributions was found for the 19 December 2003 shock, and the electric potential was found to vary smoothly on scales comparable to those of the magnetic field, in agreement with other estimates relying on electron moments. The correspondence was less satisfying for the two other shocks, indicating that some of the assumptions are not entirely appropriate to these shocks.

[38] Large-scale deviations from strict stationarity and planarity can affect the results, since an important requirement of the technique is that locations of the original and mapped distributions are sufficiently well connected by magnetic field lines and particle trajectories [Schwartz et al., 2000; Hull et al., 2000]. For instance on the Cluster separation scales some variability in the shock profiles is often observed, particularly in the foot [Horbury et al., 2001]. Such a limitation can be however in good part relaxed with the use of an appropriately located pair of satellites, as we have done for the 19 March 2005 shock. In that case, the multisatellite and single-satellite results were found similar, which is a further confirmation that stationarity and planarity are relevant here. Another possible limitation of the technique related to the large-scale structure of the shock comes from the accurate determination of de Hoffmann-Teller frame velocity (and its parallel component). This requirement should become critical for nearly perpendicular shocks, notwithstanding the fact that for these shocks the de Hoffmann-Teller velocity becomes significantly larger than the electron thermal velocity (corresponding to a high de Hoffmann-Teller electron thermal Mach number) in which case the mapping predicts highly skewed distributions which are very unlikely to be stable.

[39] On ion scales, fluctuations propagating at a small angle with respect to the shock surface (ripples) break both planarity and stationarity hypotheses and were recently found at a shock crossing on 19 December 2003 closely following the one studied in this paper by Moullard et al. [2006]. Such ripples were found in hybrid simulations to participate in the formation of a population of downstream suprathermal electrons [Burgess, 2006], but at higher energies than considered here. More dramatic large-scale effects, as well as sharper gradients on the $c / \omega_{p e}$ scale, can appear in self-reforming shocks [Krasnoselskikh et al., 2002]. In particular, simulations have shown important changes in the electric potential for such shocks [Scholer et al., 2003]. All these effects are likely to strongly affect electron dynamics, but are thought to concern essentially high-Mach number and low- $\beta$ shocks.

[40] The processes which are most likely to invalidate the assumptions the mapping and calculation of the electric potential locally in the shock front are therefore waves, localized structures or gradients on electron scales. Early research has mainly focused on current-driven microinstabilities such as the ion-acoustic or lower-hybrid drift instabilities, but there is so far a lack of clear evidence for their generation and sustainability in the shock front [see, e.g., Scudder et al., 1986b; Balikhin et al., 2005], in particular for high- $\beta$ shocks. The gyroresonant interaction of electrons with waves is nevertheless a highly efficient mean to break conservation of their adiabatic invariant and energy. Orlowski et al. [1995] have found some experimental evidence for gyro-resonant interaction of oblique suprathermal electrons with whistlers in quasi-perpendicular shock ramps. According to Veltri et al. [1990] the scattering by waves on electron-scales $\left(c / \omega_{p e}\right)$ provides a second-order effect which would "cool" electrons by make transmitted trajectories fall into the lower-energy region of phase space occupied by reflected trajectories based on guiding-center dynamics in the large-scale static fields. At least, waves are expected to smooth via pitch-angle scattering any discontinuity which may occur in the electron distribution functions near the separatrix between passing and reflected trajectories inferred from the mapping. Similarly, Vandas [2001] has argued that the shock drift acceleration mechanism, which is based on identical assumptions to those discussed here, cannot alone account for some of the most salient properties of high-energy $(>10 \mathrm{keV})$ electrons observed upstream of quasi-perpendicular shocks, and that therefore some pitch-angle scattering is likely to occur within the shock layer.

[41] Alternatively, the hypotheses can be invalidated by the presence of strong localized electric fields inside the shock layer. It has been argued by Balikhin et al. [1993] that sharp gradients (on scales of the order the electron inertial length) in the electric potential in the ramp might locally demagnetize electrons in the thermal energy range and possibly result in stronger electron heating. According to Gedalin et al. [1995] and Balikhin et al. [1998], the adiabatic invariant breakdown depends on the electric field gradients (i.e., potential curvature), and electrons with the lowest magnetic moment are the most sensitive to this demagnetization. This is supposed to occur in the foot or upstream half of the ramp, where the magnetic field is still relatively low, provided there are significantly large potential gradients on scales of a few $c / \omega_{p e}$. Furthermore, this was found to become less efficient for high- $\beta$ shocks where the thermal electrons remain strongly magnetized. Such a mechanism however concerns electrons within a limited range of gyroradii, since neither electrons with gyroradii much smaller nor much larger [Pesses, 1981; Ball and Melrose, 2001] than the ramp thickness are expected to 
break the conservation of their magnetic moments. Lembège et al. [2003] and Savoini et al. [2005] have however found that a noticeable fraction of test particle electrons thrown in PIC shock simulations follow this sort of behavior, that fraction increasing with shock nonstationary. Curiously, processes during these electrons traversal of the foot appear to play a role in enhancing the apparent demagnetization. Although the direct consequences of this phenomenon are difficult to check in the data, it is worth noting that the discrepancies between potentials from the mapping and the fluid equations as well as differences between apparent potentials for low- and high- $\mu$ electrons appear in the upstream half of the shock transition. Furthermore, they are most noticeable for the 23 January 2004 shock, which has the lowest $\beta_{e}$ and exhibits sudden and sharp gradients in the estimated electric potential. It must nevertheless be noted that results for this shock are hampered by the quite short crossing time and may suffer from time-aliasing.

[42] There are therefore several mechanisms which can break the main assumptions used to describe electron dynamics at quasi-perpendicular shocks, but they remain difficult to identify clearly. Nevertheless, at least one of them must be present, and provide additional effects to electron velocity distributions on top of the firmly established "phase space inflation" due to the large-scale stationary magnetic and electric fields. If this (these) process(es) result in an additional heating or cooling of the electrons, this implies that the electric potential estimated from a model which discards them will be respectively too large or too small. In particular, processes which shall have the greatest effect on the potential are those which affect the electrons with small pitch angles (such as ionacoustic turbulence or demagnetization of parallel electrons). To the least, one can conclude from the different values of the potentials estimated from different range of magnetic moments indicate that these processes affect differently electrons with different adiabatic invariants.

\section{Summary and Conclusion}

[43] We have used the close relationship between electron dynamics across collisionless quasi-perpendicular shocks in the drift approximation and the cross-shock electric potential in the de Hoffmann-Teller frame to derive an estimate of this potential from the measured electron distribution functions. This relationship is built upon fairly general hypotheses and simple arguments (shock planarity and stationarity, validity of the drift ordering for electrons, energy conservation in the de Hoffmann-Teller frame), and therefore is expected to be a robust one.

[44] A direct test of this relationship can be carried out by comparing the parts of the electron distribution function it allows to map from one location to another with the measured distributions. We have carried out such tests, extending previous works by considering stronger shocks, studying the dynamics of non-field aligned particles including the reflected and trapped particles, and by taking into account the overshoot in the mapping of measured distributions. The predictions were generally found to be consistent with the observations, with even remarkable agreement for a high- $\beta_{e}$ shock exhibiting relatively low electron heating (19 December 2003). For the stronger shocks (23
January 2004 and 19 March 2005), some discrepancies nevertheless appeared which can be summarized as follows:

[45] 1. In the parallel direction, the predicted velocity distribution tends to be steeper than the observed one, which results in an overestimation of the predicted distribution with respect to the observed one for the lowest-energy passing electrons, as already noticed by Scudder et al. [1986b].

[46] 2. For larger pitch-angles, the predicted distribution significantly underestimates the observed one.

[47] 3. There is a lack of symmetry in the reflected parts of the bulk of the distributions, both upstream and upstream, at least if we consistently use the same estimate of the de Hoffmann-Teller velocity as for the transmitted electrons.

[48] These results suggest that additional processes are required to smooth the discontinuities between the transmitted and reflected parts of the distributions, and to scatter field-aligned electrons (particularly the low-energy transmitted ones) to more oblique velocities. It can even be argued that such irreversible effects must always be present in order to contribute to the entropy jump across the shock. In the parallel direction these additional processes need to make the mapped distribution less steep, for example by "cooling" these electrons as suggested by Scudder et al. [1986b] and Veltri et al. [1990]. In the perpendicular direction the opposite is required, namely an extra "heating." This either suggests a single mechanism which efficiently pitch-angle scatters electrons from parallel to perpendicular directions or possibly two complementary ones.

[49] To the extent that these additional processes do not dominate the evolution of the electron distribution function through the shock, the mapping provides a way to estimate the cross-shock electric potential using less restrictive hypotheses than techniques relying on electron velocity moments, and the use of multiple satellites allows to make the hypotheses even less stringent. The estimates were found to agree quite well for two of the shocks (19 December 2003 and 19 March 2005), and the electric potential was found to change on scales comparable to those of the magnetic field in the shock layer, while displaying less oscillations downstream.

[50] However, potentials estimated by mapping electrons within a restricted range of magnetic moments have shown a dependency on the selected range which is not fully consistent with the hypotheses underlying the mapping. The apparent potential for high magnetic moment electrons (above the thermal magnetic moment) was found to generally exceed the one for lower magnetic moments, consistently with the above noted underestimation of the mapped velocity distribution with respect to the observed one for large pitch-angle electrons. This suggests that depending on their adiabatic invariant, electrons are affect differently by processes not included in the mapping.

[51] Furthermore, the moments and mapping methods disagree for the 23 January 2004 shock, the potential from the mapping exhibiting at the spin-period temporal resolution $(4 \mathrm{~s})$ significantly stronger gradients in the ramp than the magnetic field intensity. The presence of stronger de Hoffmann-Teller electric fields for this shock is consistent with the stronger discrepancies between the mapped and observed distributions but at the same time reduces the 
reliability of the potential estimated from the mapping (and from electron moments as well). It is worth noting that this shock has the smallest $\beta$ of the shocks studied in this paper, and the largest difference in proton bulk kinetic energy in the normal incidence frame, $m_{p}\left(u_{p, d}^{2}-u_{p, u}^{2}\right) / 2$, two factors which were found in statistical surveys to be best correlated with electron heating at quasi-perpendicular shocks [Thomsen et al., 1987; Schwartz et al., 1988]. The lower $\beta$ and higher Mach number shocks are therefore of high interest for a complete understanding of electron dynamics at quasi-perpendicular shocks, as stronger deviations from the usually assumed behavior are expected and could therefore better be identified. Since such deviations are expected to be closely related to small-scale phenomena yet to be clearly identified (strong gradients, small-scale structures, or wave-particle interactions), such a study shall necessarily be completed by high-resolution field data and may possibly require higher temporal resolution electron instruments.

[52] Acknowledgments. The authors thank both referees for constructive comments. They also benefited from discussions on this topic with M.A. Balikhin, D. Burgess and A.J. Hull, and wish to thank E.A. Lucek for the high-resolution FGM data used in this paper. Other FGM, CIS, and EFW data used in this study were drawn from the Cluster Prime Parameter Database and the Cluster Active Archive. Financial support from the UK PPARC is acknowledged.

[53] Amitava Bhattacharjee thanks the reviewers for their assistance in evaluating this paper.

\section{References}

Bale, S. D., et al. (2005), Quasi-perpendicular shock structure and processes, Space Sci. Rev., 118, 161-203, doi:10.1007/s11214-005-3827-0.

Balikhin, M., M. Gedalin, and A. Petrukovich (1993), New mechanism for electron heating in shocks, Phys. Rev. Lett., 70, 1259-1262.

Balikhin, M., V. V. Krasnosel'skikh, L. J. C. Woolliscroft, and M Gedalin (1998), A study of the dispersion of the electron distribution in the presence of E and B gradients: Application to electron heating at quasiperpendicular shocks, J. Geophys. Res., 103, 2029-2040.

Balikhin, M., S. Walker, R. Treumann, H. Alleyne, V. Krasnoselskikh, M. Gedalin, M. Andre, M. Dunlop, and A. Fazakerley (2005), Ion sound wave packets at the quasiperpendicular shock front, Geophys. Res. Lett. 32, L24106, doi:10.1029/2005GL024660.

Ball, L., and D. B. Melrose (2001), Shock Drift Acceleration of Electrons, Publ. Astron. Soc. Aust., 18, 361-373, doi:10.1071/AS01047.

Balogh, A., et al. (2001), The Cluster Magnetic Field Investigation: Overview of in-flight performance and initial results, Ann. Geophys., 19, $1207-1217$.

Brizard, A. J., and T. S. Hahm (2007), Foundations of nonlinear gyrokinetic theory, Rev. Mod. Phys., 79, 421-468, doi:10.1103/RevModPhys.79.421.

Burgess, D. (2006), Simulations of electron acceleration at collisionless shocks: The effects of surface fluctuations, Astrophys. J., 653, 316324, doi:10.1086/508805.

Décréau, P., et al. (2001), Early results from the Whisper instrument on Cluster: An overview, Ann. Geophys., 19, 1241-1258.

Dunlop, M. W., and T. I. Woodward (2000), Multi-spacecraft discontinuity analysis: orientation and motion, in Analysis Methods for Multi-Spacecraft Data, ISSI Sci. Rep., vol. SR-001, edited by G. Paschmann and P. Daly, pp. 271-306, Springer, New York.

Feldman, W. C., R. C. Anderson, S. J. Bame, S. P. Gary, J. T. Gosling, D. J. McComas, M. F. Thomsen, G. Paschmann, and M. M. Hoppe (1983), Electron velocity distributions near the Earth's bow shock, J. Geophys. Res., 88, 96-110.

Fitzenreiter, R. J., K. W. Ogilvie, S. D. Bale, and A. F. Viñas (2003), Modification of the solar wind electron velocity distribution at interplanetary shocks, J. Geophys. Res., 108(A12), 1415, doi:10.1029/ 2003JA009865.

Formisano, V. (1982), Measurement of the potential drop across the Earth's collisionless bow shock, Geophys. Res. Lett., 9, 1033-1036.

Gedalin, M., and E. Griv (1999), Role of overshoots in the formation of the downstream distribution of adiabatic electrons, J. Geophys. Res., 104, $14,821-14,826$
Gedalin, M., K. Gedalin, M. Balikhin, and V. Krasnosselskikh (1995), Demagnetization of electrons in the electromagnetic field structure, typical for quasi-perpendicular collisionless shock front, J. Geophys. Res. $100,9481-9488$.

Goodrich, C. C., and J. D. Scudder (1984), The adiabatic energy change of plasma electrons and the frame dependence of the cross-shock potential at collisionless magnetosonic shock waves, J. Geophys. Res., 89, 66546662 .

Gustafsson, G., et al. (1997), The Electric Field and Wave Experiment for the Cluster Mission, Space Sci. Rev., 79, 137-156.

Horbury, T. S., P. J. Cargill, E. A. Lucek, A. Balogh, M. W. Dunlop, T. M. Oddy, C. Carr, P. Brown, A. Szabo, and K.-H. Fornaçon (2001), Cluster magnetic field observations of the bowshock: Orientation, motion and structure, Ann. Geophys., 19, 1399-1409.

Hull, A. J., J. D. Scudder, L. A. Frank, W. Paterson, and M. G. Kivelson (1998), Electron heating and phase space signatures at strong and weak quasi-perpendicular shocks, J. Geophys. Res., 103, 2041-2054.

Hull, A. J., J. D. Scudder, R. J. Fitzenreiter, K. W. Ogilvie, J. A. Newbury, and C. T. Russell (2000), Electron temperature and de Hoffmann-Teller potential change across the Earth's bow shock: New results from ISEE 1, J. Geophys. Res., 105, 20,957-20,972.

Hull, A. J., J. D. Scudder, D. E. Larson, and R. Lin (2001), Electron heating and phase space signatures at supercritical, fast mode shocks, J. Geophys. Res., 106, 15,711-15,734.

Johnstone, A. D., et al. (1997), Peace: a Plasma Electron and Current Experiment, Space Sci. Rev., 79, 351-398.

Krasnoselskikh, V. V., B. Lembège, P. Savoini, and V. V. Lobzin (2002), Nonstationarity of strong collisionless quasiperpendicular shocks: Theory and full particle numerical simulations, Phys. Plasmas, 9, 1192-1209.

Lee, M. A., V. D. Shapiro, and R. Z. Sagdeev (1996), Pickup ion energization by shock surfing, J. Geophys. Res., 101, 4777-4790.

Lembège, B., P. Savoini, M. Balikhin, S. Walker, and V. Krasnoselskikh (2003), Demagnetization of transmitted electrons through a quasi-perpendicular collisionless shock, J. Geophys. Res., 108(A6), 1256 doi:10.1029/2002JA009288.

Leroy, M. M., and A. Mangeney (1984), A theory of energization of solar wind electrons by the Earth's bow shock, Ann. Geophys., 2, $448-456$

Lobzin, V. V., V. V. Krasnoselskikh, S. J. Schwartz, I. Cairns, B. Lefebvre, P. Décréau, and A. Fazakerley (2005), Generation of downshifted oscillations in the electron foreshock: A loss-cone instability, Geophys. Res. Lett., 32, L18101, doi:10.1029/2005GL023563.

Montgomery, M. D., J. R. Asbridge, and S. J. Bame (1970), Vela 4 Plasma Observations Near the Earth's Bow Shock, J. Geophys. Res., 75, $1217-$ 1231

Moullard, O., D. Burgess, T. S. Horbury, and E. A. Lucek (2006), Ripples observed on the surface of the Earth's quasi-perpendicular bow shock, J. Geophys. Res., 111, A09113, doi:10.1029/2005JA011594.

Orlowski, D. S., C. T. Russell, D. Krauss-Varban, N. Omidi, and M. F. Thomsen (1995), Damping and spectral formation of upstream whistlers, J. Geophys. Res., 100, 17,117-17,128.

Peredo, M., J. A. Slavin, E. Mazur, and S. A. Curtis (1995), Three-dimensional position and shape of the bow shock and their variation with Alfvenic, sonic and magnetosonic Mach numbers and interplanetary magnetic field orientation, J. Geophys. Res., 100, 7907-7916.

Pesses, M. E. (1981), On the conservation of the first adiabatic invariant in perpendicular shocks, J. Geophys. Res., 86, 150-152.

Rème, H., et al. (2001), First multispacecraft ion measurements in and near the Earth's magnetosphere with the identical Cluster ion spectrometry (CIS) experiment, Ann. Geophys., 19, 1303-1354.

Sagdeev, R. Z. (1966), Cooperative phenomena and shock waves in collisionless plasmas, Rev. Plasma Phys., 4, $23-91$.

Savoini, P., B. Lembège, V. Krasnosselskhik, and Y. Kuramitsu (2005), Under and over-adiabatic electrons through a perpendicular collisionless shock: Theory versus simulations, Ann. Geophys., 23, 3685-3698.

Scholer, M. I. Shinohara, and S. Matsukiyo (2003), Quasi-perpendicular shocks: Length scale of the cross-shock potential, shock reformation, and implication for shock surfing, J. Geophys. Res., 108(A1), 1014, doi:10.1029/2002JA009515.

Schwartz, S. J. (2000), Shock and discontinuity normals, mach numbers, and related parameters, in Analysis Methods for Multi-Spacecraft Data, ISSI Sci. Rep., vol. SR-001, edited by G. Paschmann and P. Daly, pp. 249-270, Springer, New York.

Schwartz, S. J., M. F. Thomsen, S. J. Bame, and J. Stansberry (1988), Electron heating and the potential jump across fast mode shocks, J. Geophys. Res., 93, 12,923-12,931.

Schwartz, S. J., P. W. Daly, and A. N. Fazakerley (2000), Multi-spacecraft analysis of plasma kinetics, in Analysis Methods for Multi-Spacecraft Data, ISSI Sci. Rep., vol. SR-001, edited by G. Paschmann and P. Daly, pp. 159-183, Springer, New York. 
Scudder, J. D. (1987), The field-aligned flow approximation for electrons within layers possessing a normal mass flux - A corollary to the deHoffmann-Teller theorem, J. Geophys. Res., 92, 13,447-13,455.

Scudder, J. D. (1995), A review of the physics of electron heating at collisionless shocks, Adv. Space Res., 15, 181-223.

Scudder, J. D., A. Mangeney, C. Lacombe, C. C. Harvey, and T. L. Aggson (1986a), The resolved layer of a collisionless, high beta, supercritical, quasi-perpendicular shock wave: 2 . Dissipative fluid electrodynamics, J. Geophys. Res., 91, 11,053-11,073.

Scudder, J. D., A. Mangeney, C. Lacombe, C. C. Harvey, and C. S. Wu (1986b), The resolved layer of a collisionless, high beta, supercritical, quasi-perpendicular shock wave: 3. Vlasov electrodynamics, J. Geophys Res., 91, 11,075-11,097.

Thomsen, M. F., J. A. Stansberry, S. J. Bame, J. T. Gosling, and M. M. Mellott (1987), Strong electron heating at the earth's bow shock, J. Geophys. Res., 92, 10,119-10,124.

Vandas, M. (2001), Shock drift acceleration of electrons: A parametric study, J. Geophys. Res., 106, 1859-1872, doi:10.1029/2000JA900128.

Veltri, P., A. Mangeney, and J. Scudder (1990), Electron heating in quasiperpendicular shocks: A Monte Carlo study, J. Geophys. Res., 95 , $14,939-14,959$
Walker, S., H. Alleyne, M. Balikhin, M. André, and T. Horbury (2004), Electric field scales at quasi-perpendicular shocks, Ann. Geophys., 22, $2291-2300$

Wu, C. S. (1984), A fast Fermi process - Energetic electrons accelerated by a nearly perpendicular bow shock, J. Geophys. Res., 89, 8857-8862.

Wygant, J. R., M. Bensadoun, and F. S. Mozer (1987), Electric field measurements at subcritical, oblique bow shock crossings, J. Geophys. Res., 92, 11,109-11,121.

Zank, G. P., H. L. Pauls, I. H. Cairns, and G. M. Webb (1996), Interstellar pickup ions and quasi-perpendicular shocks: Implications for the termination shock and interplanetary shocks, J. Geophys. Res., 101, 457-478.

P. Décréau, LPCE, CNRS and University of Orléans, $3 \mathrm{~A}$ av de la Recherche Scientifique, 45071 Orléans, France. (pierrette.decreau@ cnrs-orleans.fr)

A. F. Fazakerley, Mullard Space Science Laboratory, University College London, Holmbury St. Mary, Dorking, Surrey RH5 6NT, UK. (anf@mssl. ucl.ac.uk)

B. Lefebvre and S. J. Schwartz, Space and Atmospheric Physics, Blackett Laboratory, Imperial College London, London SW7 2BW, UK. (b.lefebvre@imperial.ac.uk; s.schwartz@imperial.ac.uk) 